NBSIR 77-1222

\title{
Reproducibility of the Radiant Panel Test Method (ASTM E 162-67) Using Polyurethane Foam, Neoprene, and Hardboard Specimens
}

T. G. Lee

Center for Fire Research

Institute for Applied Technology

National Bureau of Standards

Washington, D.C. 20234

March 1977

Final Report

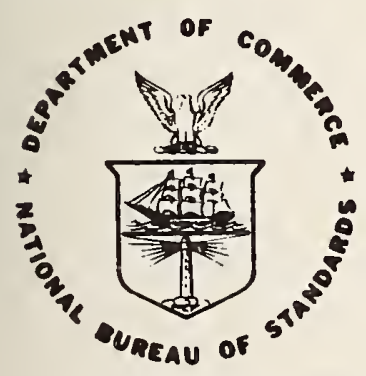

U. S. DEPARTMENT OF COMMERCE

NATIONAL BUREAU OF STANDARDS 
. 
NBSIR 77-1222

REPRODUCIBILITY OF THE RADIANT

PANEL TEST METHOD (ASTM E

162-67) USING POLYURETHANE

FOAM, NEOPRENE, AND HARDBOARD

SPECIMENS

T. G. Lee

Center for Fire Research

Institute for Applied Technology

National Bureau of Standards

Washington, D.C. 20234

March 1977

Final Report

U.S. DEPARTMENT OF COMMERCE, Juanita M. Kreps, Secretary

Dr. Betsy Ancker-Johnson, Assistant Secretary for Science and Technology

NATIONAL BUREAU OF STANDARDS, Ernest Ambler, Acting Director 
CONTENTS

Page

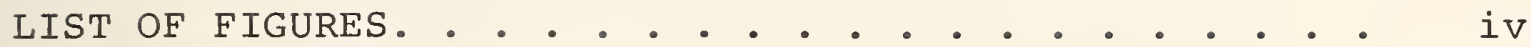

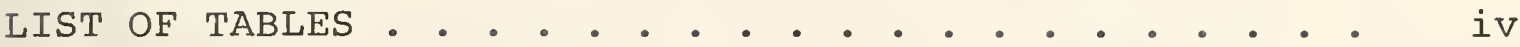

Abstract . . . . . . . . . . . . . . . . . . 1

1. INTRODUCTION • . . . . . . . . . . . . . . . . 1

2. PARTICIPANTS • . . . . . . . . . . . . . . . . . 2

3. TESTING PROCEDURES • . . . . . . . . . . . . . . 3

3.1. Standard Method. . . . . . . . . . . 3

3.2. Modification of Testing Procedures . . . . . 4

4. TEST MATERIALS • . . . • • . . . • . • . 5

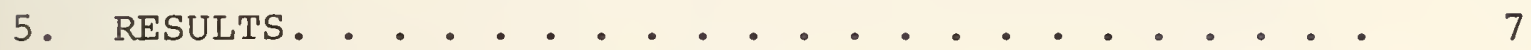

5.1. Survey of Operating Characteristics. . . . 7

5.2. Test Results . . . . . . . . . . . 7

6. DISCUSSION • • . . . . . . . . . . . . . . 11

6.1. Variability and Source of Errors . . . . . . 11

6.2. Effect of Preheating Urethane. . . . . . . 22

6.3. Rank Ordering of Laboratories. . . . . . . 22

6.4. Effect of Procedure Modifications. . . . . 23

6.5. Analysis of Data . . . . . . . . . 23

7. SUMMARY AND CONCLUSIONS • • • • • • • • • • . . . 25

8. ACKNOWLEDGMENTS • • . . • . . . . . . . . 26

9. REFERENCES • . . . . . . . . . . . . . . 26

APPENDIX A. DETAILS ON TEST APPARATUS AND PROCEDURE

MODIFICATIONS OF THE RADIANT PANEL METHOD 28

APPENDIX B. SAMPLE CALCULATIONS FOR HEAT INPUT RATE

CORRECTION USED IN CALIBRATION OF

RADIANT PANEL STACK THERMOCOUPLES • • • . 31

APPENDIX BI. SI CONVERSION UNITS • . . 33

APPENDIX C. DEFINITION OF TERMS USED IN THE STATISTICAL

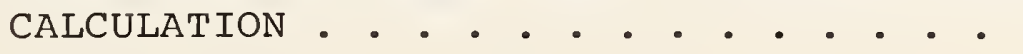


Figure 1. Construction and Location of the Modified Pilot Ignitor . . . . . . . . . . 6

Figure 2. Data Sheet of Lab O for Hardboard A . . . 14

Figure 3. Plots of Flame Fronts of Hardboard A by

$\mathrm{Lab} O$. . . . . . . . . . . . . 15

Figure 4. Data Sheet of Lab E for Neoprene C. • . . 16

Figure 5. Plots of Flame Fronts of Neoprene C by

Lab E. . . . . . . . . . . . 17

Figure 6. Data Sheet of Lab I for Polyurethane B. . . 18

Figure 7. Plots of Flame Front of Polyurethane B by Lab I. . . . . . . . . . . . . 19

Figure 8. Correlation Between $Q$ and $1 / \beta$ for All Labs. 20

Figure 9. Mean I of Each Lab versus Mean $I_{s}$ for

\section{LIST OF TABLES}

Table 1. Calibration and Operating Conditions for Participating Laboratories . . . . . . .

Table 2. Test Results for Each Specimen and Laboratory

Table 3. Effect of Preheating on Flame-spread Index of Urethane Foam B. . . . . . . . . 12

Table 4. Statistical Results. . . . . . . . . . . 13 
REPRODUCIBILITY OF THE RADIANT PANEL TEST METHOD

(ASTM E 162-67) USNG POLYURETHANE FOAM, NEOPRENE, AND HARDBOARD SPECIMENS

T. G. Lee

Abstract

Interlaboratory evaluation of the Radiant Panel Method (ASTM E 162-67) for flame spread testing of two flexible foam and one hardboard specimens was made. Results obtained by 13

laboratories, based on 4 replicate tests, showed that the between-lab coefficient of variation on the flame-spread index ( $\left.I_{S}\right)$ was $21 \%$ for Hardboard A, 35\% for Urethane B and 45\% for Neoprene C. The within-lab coefficient of variation for the Hardboard was 9.9\%. The higher variability of the results for the foam materials was caused by the rapid melting of the Urethane $B$ and unstable flame front of the Neoprene $C$ specimens during the tests. An important source of error for some laboratories was in the determination of $\beta$, the calibration constants, and the inappropriate use of base stack temperature correction. Statistics on the reproducibility of the flame-spread factor $\left(\mathrm{F}_{\mathrm{S}}\right)$, heat evolution $(Q)$, and $I$ are also given. A new ${ }^{S}$ pilot burner and other modifications of the method were found useful.

Key words: ASTM E 162; coefficient of variation; flame spread tests; flexible polyurethane; hardboard; interlaboratory evaluation; neoprene; radiant panel test; round robin; test method.

\section{INTRODUCTION}

Data on the reproducibility of a standard test method for various types of specimens are important to the testing laboratories as well as to the material producers. These data often form the basis for adjusting the testing procedures and calibration techniques employed by the testing laboratories. The data on the repeatability of the method for a given type of material of known variability would also allow the material producer to determine whether its test results reflect a real change of the material property or merely the uncertainty of the method as it applies to this type of material. 
Test methods and procedures are generally designed to measure a particular material property for a limited range of materials. Precision statements on the method based on tests of these materials are usually included in the method or documented in the open literature. However, if the test method were to be used for a different type of material only marginally compatible with the original method, new studies including test method modification should be made to determine the reproducibility.

The Radiant Panel Test, ASTM E 162-67 [1-3] ${ }^{1}$ was adopted by the American Society of Testing and Materials in 1967. It was designed to measure surface flame spread of non-melting or very slow-melting solid materials. However, this method has also been used recently for testing flexible foam and other thermoplastic materials.

To ascertain the reproducibility of the method as practiced by major testing laboratories on the flexible foam materials, an interlaboratory evaluation, the so-called "round-robin" study, was made. This paper reports the results of the pilot phase of the study suggested originally by the Flexible Foam Flammability Task Group of the Society of Plastic Industry (SPI). The aim of this preliminary phase was to pinpoint and correct some of the problems, which laboratory operators may encounter, prior to the main study and to examine the effectiveness of the modifications introduced for testing thermoplastic foam materials.

An unpublished study in 1958 by Gross [4], based on radiant panel tests of four acoustic tile materials by eight laboratories, showed that the use of uncalibrated radiation pyrometers to monitor the panel output was the primary cause of large systematic biases observed in the test results. The present study used calibrated pyrometers.

Since no published study on the reproducibility of this method is available, the results of this limited work should be useful to the estimated 40 laboratories who are using the method for routine testing.

\section{PARTICIPANTS}

This report is based on the results from all 13 laboratories which collaborated in this joint study. The laboratories are:

${ }^{1}$ Numbers in brackets refer to the literature references listed at the end of this paper. 
Armstrong Cork Company (John Crawford)

E. I. duPont de Nemours and Company (R. L. Jones)

Federal Aviation Administration (Eldon Nicholas)

Lawrence Livermore Laboratory (James Gaskill)

$M \& T$ Chemical (I. Touval)

Mobay Chemical (W. J. Eicher)

National Bureau of Standards (Roland Willard)

National Research Council of Canada (P. Huot)

Olin Corporation (Margot Raetz)

Stauffer Chemical Company (Richard Cope, Jr.)

Toyad Corporation (Ralph Morford)

U. S. Bureau of Mines (Hary Verakis)

U. S. Testing Company (J.E. Fuller)

These laboratories are identified in this report by code letters only.

\section{TESTING PROCEDURES}

\subsection{Standard Method}

The apparatus and procedures of the standard radiant panel method have been described in detail [1-3]. Essentially, the radiant panel consists of a cast iron frame enclosing a $30 \mathrm{~cm}$ wide by $46 \mathrm{~cm}$ high porous refractory material. The panel is mounted in a vertical plane, and a premixed air-gas mixture supplied from the rear is burned in intimate contact with the refractory surface to provide a radiant heat source. The energy output of the panel, as measured by a radiation pyrometer, is equivalent to a blackbody temperature of $670{ }^{\circ} \mathrm{C}$. A stack, placed under an exhaust hood and above the test specimen, receives the smoke and hot products of combustion.

For the test, the $15-$ by $46-\mathrm{cm}$ specimen, backed by a sheet of asbestos millboard, was placed in a metal holder and mounted on a supporting frame. The specimen faced and inclined 30 degrees to the radiant panel such that the specimen was closer to the panel at the top. A pilot igniter, fed by an air-acetylene mixture, served both to initiate flaming at the upper edge of the test specimen and to ignite combustible gases rising from the specimen. Observations were then made of the progress of the flame front, the occurrence of intermittent flaming as well as the temperature rise of the stack thermocouples. The test duration was 15 minutes or until sustained flaming had traversed downward the entire $46-\mathrm{cm}$ length of specimen, whichever time was less.

The flame-spread index, I ${ }^{\prime}$, was computed as the product of the flame-spread factor, $F_{S^{\prime}}{ }^{\prime}$ and the heat evolution, $Q$, or: 


$$
I_{S}=F_{S} Q
$$

where

$$
F_{S}=1+\frac{1}{t_{3}}+\frac{1}{t_{6}-t_{3}}+\frac{1}{t_{9}-t_{6}}+\frac{1}{t_{12}-t_{9}}+\frac{1}{t_{15}-t_{12}} \text {. }
$$

The symbols $t_{3} \cdot t_{15}$ correspond to the times in minutes from specimen exposure until arrival of the flame front at a position $7.6 \mathrm{~cm}$ ( 3 in), $15.2 \mathrm{~cm}$ ( 6 in) . . $38 \mathrm{~cm}$ (15 in), respectively, along the length of the specimen. The heat of evolution, Q is expressed as

$$
Q=0.1 \Delta \mathrm{T} / \beta
$$

where 0.1 is an arbitrary constant. $\Delta \mathrm{T}$ is the observed maximum stack thermocouple temperature rise above ambient for the specimen minus the maximum temperature rise observed with a thick asbestos-cement board substituted for the specimen. The $\beta$ is a calibration constant representing the maximum stack thermocouple temperature rise per unit heat input rate using a calibrating diffusion-type gas burner, placed near the top of an asbestos-cement board specimen during normal operation of the radiant panel. .

Prior to the test, all specimens and composite assemblies were conditioned by placing them in an oven at $60{ }^{\circ} \mathrm{C}$ for a 24-hour period and then allowing them to reach moisture equilibrium (constant weight) at $23^{\circ} \mathrm{C}$ and at relative humidities of $50+5 \%$

\subsection{Modification of Testing Procedures}

A source of error in the use of standard test methods is the interpretation and clear understanding of the written procedures. Thus, a meeting of potential participants to discuss areas of ambiguity and needed changes is always desirable prior to the testing program. Interested participants with radiant panel facilities from 20 laboratories met to discuss and standardize the calibration and test procedures. Some modifications and clarifications of the published ASTM E 162-67 standard were adopted (see appendix A). These included: a new pilot igniter, thermocouple cleaning methods, aluminum foil wrapping for the non-burning surfaces of the specimen, and the use of screening to help retain the melting specimen in the holder during testing. 
The new pilot burner was necessary because the original ignitor or burner was frequently extinguished by combustion products laden with flame inhibitor from certain types of test specimens. The new igniter consisting of a length of two-hole porcelain tubing and using premixed air-acetylene, was found to be stable and immune to "flame-out." Details of its construction, location, and flow rate are shown in figure 1 .

The thermocouple bank at the top of the stack needed periodic cleaning to eliminate heavy soot deposits which affected the time-constant of the thermocouples. To assure uniformity, all laboratories agreed to clean the thermocouples prior to each run. An air jet from pressurized tubing inserted from the side of the stack slightly above the thermocouples may be used to expedite the cleaning operation.

To avoid excessive dripping of melted specimen and subsequent cleanup, aluminum foil was used to wrap the back and sides of the specimen before insertion into the sample holder. Use of the wrapper also improved the test repeatability. Wire screen, about $25 \mathrm{~mm}$ mesh, placed over the entire exposed surface of the specimen retarded the dripping and improved test repeatability.

Other modifications and interpretations detailed in appendix $A$ were also adopted by the participating laboratories for this study. With the exception of these modifications, the tests otherwise followed closely the procedures given in the standard method.

\section{TEST MATERIALS}

Three different types of specimens were used for the preliminary study. The polyurethane and neoprene materials selected represent two of the most common flexible foams in use today. Hardboard, a common building material, was included to permit comparison of a material having a welldefined flame front and considered an "ideal" specimen for the test method. The "non-ideal" polyurethane tends to melt and flow at flaming temperature and is complicated by a fast-moving and difficult-to-interpret flame front. Neoprene on the other hand, does not melt; but it emits copious smoke including the principal flame-inhibitor $\mathrm{HCl}$, which frequently extinguished the pilot flame of the old pilot ignitor. Periodic ignition and flashing at different positions of the specimen during testing also make the flame front difficult to follow.

Test materials were obtained from commercial sources without special control of uniformity. Specimens were cut 


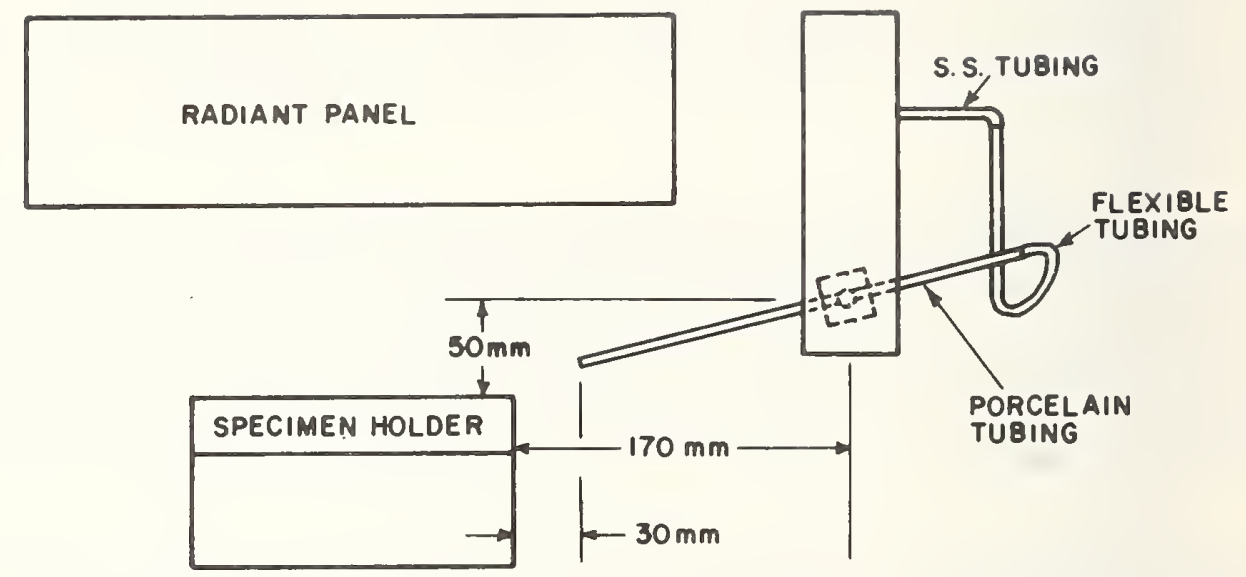

\section{TOP VIEW}

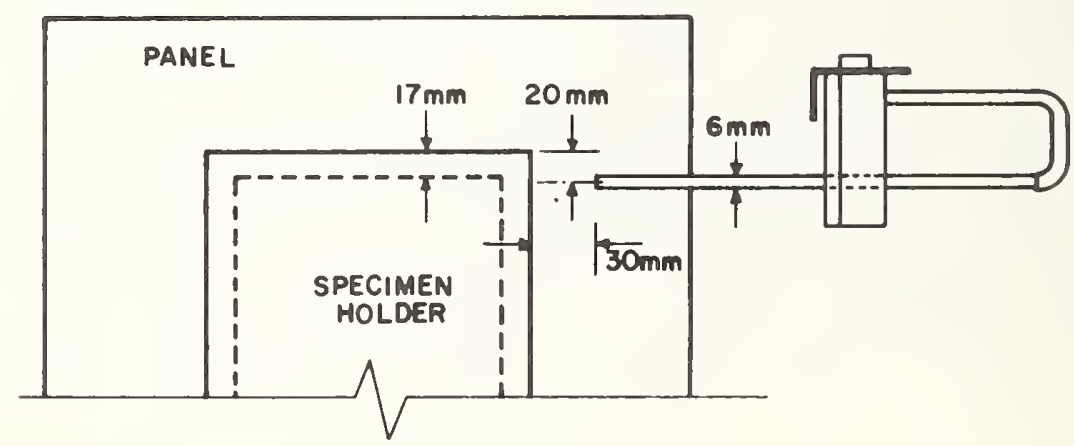

\section{SIDE VIEW}

2-HOLE PORCELAIN TUBING

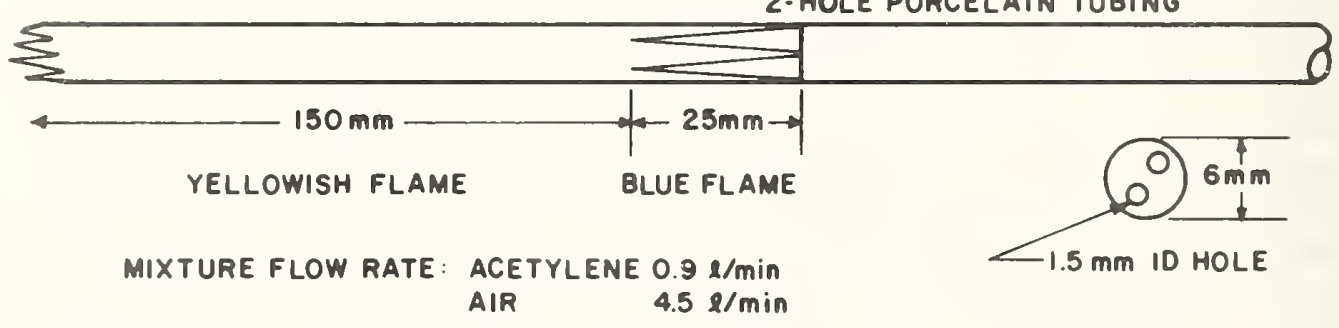

\section{PILOT FLAME}

Figure 1. Construction and Location of the Modified Pilot Ignitor 
from the same batch, randomized and numbered before distribution. The coefficient of variation of the density of the two foam materials, 100 by 100 by $25 \mathrm{~mm}$ in size, was less than +3 percent based on 10 replicate measurements. Each laboratory received four specimens of each of the three materials, and was requested to randomize the testing by following a random sequence supplied by NBS. The materials were:

\begin{tabular}{|c|c|c|}
\hline Material & $\begin{array}{l}\text { Thickness } \\
\text { (mm) }\end{array}$ & $\begin{array}{l}\text { Measured Density } \\
\left(\mathrm{g} / \mathrm{cm}^{3}\right)\end{array}$ \\
\hline Hardboard A & 8.3 & 1.05 \\
\hline $\begin{array}{l}\text { Polyurethane Foam B } \\
\text { (open cell) }\end{array}$ & 25 & 0.06 \\
\hline $\begin{array}{l}\text { Neoprene Foam C } \\
\text { (closed cell) }\end{array}$ & 25 & 0.19 \\
\hline
\end{tabular}

\section{RESULTS}

\subsection{Survey of Operating Characteristics}

Table 1 is a compilation of the operating conditions and calibration test results from each laboratory. The higher (gross) heating values of the calibration fuel (see appendix B), determined under the temperature, pressure and moisture conditions of measurement, and the resulting $\beta$ are listed. Results of the latest test on the NBS standard reference material SRM $1002 \mathrm{~b}$ are also given. The maximum stack temperature rise, $\Delta \mathrm{T}_{\mathrm{m}}$, based on the average of four runs for each of three test materials and the base temperature corrections for the asbestos-cement board, $\Delta \mathrm{T}_{\mathrm{C}}$, used in calculating the net temperature rise for the material are included on table 1. The base temperature corrections are a source of error to be discussed later.

Typical base stack temperature, type of gas used in the panel and pyrometer distance to the panel are also listed in table 1 for each laboratory.

\subsection{Test Results}

The reported data on flame-spread factor, $F_{S}$; heat evolution, Q; and flame-spread index, $I_{S}$, for all the specimens and laboratories are tabulated in table 2. These specimens were conditioned in accordance with the standard procedure. 


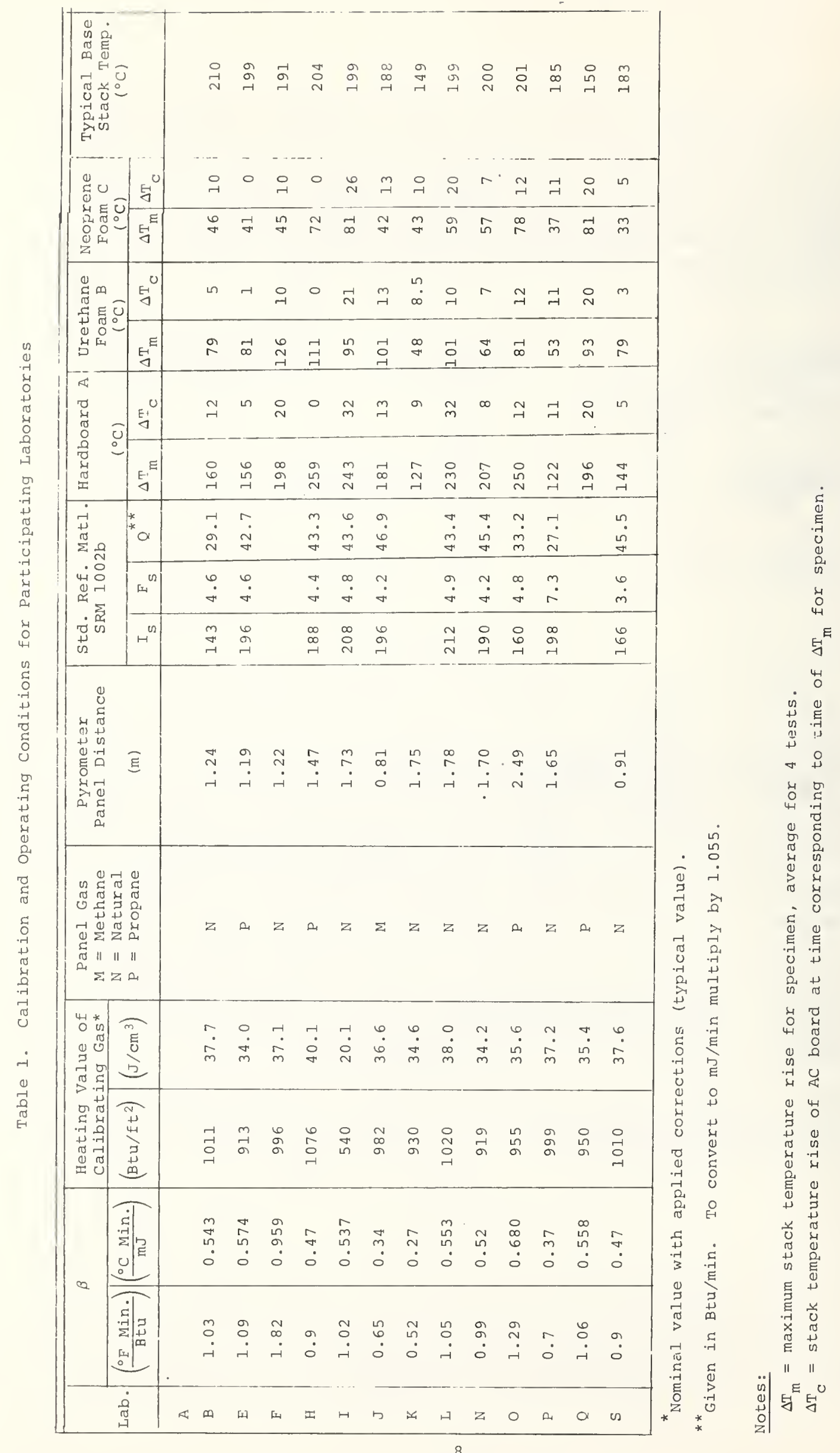


Table 2. Test Results for Each Specimen and Iaboratory

\begin{tabular}{|c|c|c|c|c|c|c|c|c|c|}
\hline Lab. & $\mathrm{F}_{\mathrm{S}}$ & $Q$ & $I_{S}$ & $\mathrm{~F}_{\mathrm{S}}$ & $Q$ & $I_{S}$ & $\mathrm{~F}_{\mathrm{S}}$ & $Q$ & $I_{S}$ \\
\hline B & $\begin{array}{l}4.6 \\
4.7 \\
5.1 \\
4.8\end{array}$ & $\begin{array}{l}27.9 \\
25.2 \\
23.8 \\
26.5 \\
.\end{array}$ & $\begin{array}{r}127.0 \\
119.0 \\
121.0 \\
127.0 \\
\quad 124\end{array}$ & $\begin{array}{l}25.5 \\
34.5 \\
37.7 \\
26.2\end{array}$ & $\begin{array}{l}11.8 \\
11.7 \\
15.9 \\
12.5\end{array}$ & $\begin{array}{r}302.0 \\
401.0 \\
601.0 \\
327.0 \\
408\end{array}$ & $\begin{array}{l}1.7^{*} \\
2.6 \\
2.7 \\
2.3\end{array}$ & $\begin{array}{l}6.5 \\
5.9 \\
6.1 \\
5.8\end{array}$ & $\begin{array}{r}11.0 \\
16.0 \\
17.0 \\
13.0 \\
14\end{array}$ \\
\hline$E$ & $\begin{array}{l}4.8 \\
5.6 \\
5.0 \\
5.0\end{array}$ & $\begin{array}{l}28.8 \\
26.9 \\
20.8 \\
23.8\end{array}$ & $\begin{array}{r}138.0 \\
151.0 \\
104.0 \\
119.0 \\
128\end{array}$ & $\begin{array}{l}36.1 \\
35.8 \\
37.0 \\
35.8\end{array}$ & $\begin{array}{l}13.9 \\
11.7 \\
13.1 \\
13.9\end{array}$ & $\begin{array}{r}507.0 \\
419.0 \\
485.0 \\
498.0 \\
476\end{array}$ & $\begin{array}{l}5.3^{E} \\
7.7^{2} \\
7.0 \\
7.0\end{array}$ & $\begin{array}{l}6.6 \\
6.3 \\
6.0 \\
5.4\end{array}$ & $\begin{array}{r}35.0 \\
49.0 \\
42.0 \\
38.0 \\
41\end{array}$ \\
\hline $\mathrm{H}$ & $\begin{array}{l}4.5 \\
4.6 \\
4.5 \\
4.6\end{array}$ & $\begin{array}{l}27.2 \\
29.4 \\
28.9 \\
29.2\end{array}$ & $\begin{array}{r}122.0 \\
136.0 \\
131.0 \\
133.0 \\
131\end{array}$ & $\begin{array}{l}43.9 \\
31.9 \\
40.0 \\
36.3\end{array}$ & $\begin{array}{l}15.5 \\
12.8 \\
11.1 \\
10.0\end{array}$ & $\begin{array}{r}682.0 \\
407.0 \\
444.0 \\
362.0 \\
474\end{array}$ & $\begin{array}{l}1.7 \\
4.5 \\
1.8 \\
1.7\end{array}$ & $\begin{array}{l}8.7 \\
9.1 \\
7.6 \\
6.7\end{array}$ & $\begin{array}{r}15.0 \\
41.0 \\
13.0 \\
12.0 \\
20\end{array}$ \\
\hline I & $\begin{array}{l}4.5 \\
4.5 \\
4.9 \\
4.4\end{array}$ & $\begin{array}{l}35.8 \\
36.3 \\
40.7 \\
36.3\end{array}$ & $\begin{array}{r}161.0 \\
164.0 \\
199.0 \\
158.0 \\
171\end{array}$ & $\begin{array}{l}30.9 \\
37.8 \\
40.3 \\
39.9\end{array}$ & $\begin{array}{l}14.7 \\
13.2 \\
11.3 \\
13.2\end{array}$ & $\begin{array}{r}456.0 \\
501.0 \\
454.0 \\
529.0 \\
485\end{array}$ & $\begin{array}{l}2.5^{\mathrm{E}} \\
3.5 \\
7.5 \\
3.2\end{array}$ & $\begin{array}{r}10.3 \\
9.3 \\
10.8 \\
8.8\end{array}$ & $\begin{array}{r}26.0 \\
33.0 \\
81.0 \\
28.0 \\
42\end{array}$ \\
\hline $\mathrm{K}$ & $\begin{array}{l}4.4 \\
4.8 \\
4.9 \\
5.6\end{array}$ & $\begin{array}{l}44.5 \\
41.1 \\
40.7 \\
41.5\end{array}$ & $\begin{array}{r}196.0 \\
197.0 \\
199.0 \\
232.0 \\
206\end{array}$ & $\begin{array}{l}32.0 \\
43.2 \\
36.5 \\
38.2\end{array}$ & $\begin{array}{l}19.7 \\
13.9 \\
17.3 \\
16.4\end{array}$ & $\begin{array}{r}617.0 \\
603.0 \\
632.0 \\
626.0 \\
619\end{array}$ & & & \\
\hline $\mathrm{L}$ & $\begin{array}{l}4.3 \\
4.1 \\
4.2 \\
4.2\end{array}$ & $\begin{array}{l}34.4 \\
34.4 \\
35.8 \\
33.2\end{array}$ & $\begin{array}{r}149.0 \\
141.0 \\
151.0 \\
140.0 \\
145\end{array}$ & $\begin{array}{l}47.4 \\
37.7 \\
36.2 \\
50.8\end{array}$ & $\begin{array}{l}15.2 \\
14.7 \\
17.1 \\
15.2\end{array}$ & $\begin{array}{r}722.0 \\
555.0 \\
620.0 \\
775.0 \\
668\end{array}$ & $\begin{array}{l}4.1 \\
3.9 \\
3.6 \\
7.2\end{array}$ & $\begin{array}{l}7.0 \\
7.0 \\
6.3 \\
6.5\end{array}$ & $\begin{array}{r}29.0 \\
27.0 \\
23.0 \\
47.0 \\
32\end{array}$ \\
\hline $\mathrm{N}$ & $\begin{array}{l}4.6 \\
4.2 \\
4.8 \\
4.3\end{array}$ & $\begin{array}{l}35.0 \\
37.3 \\
33.9 \\
34.2\end{array}$ & $\begin{array}{r}160.0 \\
157.0 \\
162.0 \\
146.0 \\
156\end{array}$ & $\begin{array}{l}41.5 \\
40.1 \\
41.9 \\
43.8\end{array}$ & $\begin{array}{r}12.2 \\
9.0 \\
12.3 \\
8.6\end{array}$ & $\begin{array}{r}506.0 \\
361.0 \\
515.0 \\
376.0 \\
440\end{array}$ & $\begin{array}{l}5.1^{\mathrm{E}} \\
1.7 \\
6.1 \\
4.6\end{array}$ & $\begin{array}{l}8.4 \\
7.3 \\
8.1 \\
7.5\end{array}$ & $\begin{array}{r}43.0 \\
13.0 \\
49.0 \\
34.0 \\
35\end{array}$ \\
\hline
\end{tabular}


Table 2. Test Results for Each Specimen and Laboratory (cont'd)

\begin{tabular}{|c|c|c|c|c|c|c|c|c|c|}
\hline \multirow{2}{*}{ Lab. } & \multicolumn{3}{|c|}{ Hardboard A } & \multicolumn{3}{|c|}{ Urethane B } & \multicolumn{3}{|c|}{ Neoprene C } \\
\hline & $\mathrm{F}_{\mathrm{S}}$ & $Q$ & $I_{S}$ & $\mathrm{~F}_{\mathrm{S}}$ & $\mathrm{Q}$ & $I_{s}$ & $\mathrm{~F}_{\mathrm{S}}$ & $Q$ & $I_{S}$ \\
\hline 0 & $\begin{array}{l}4.8 \\
5.0 \\
4.8 \\
5.2\end{array}$ & $\begin{array}{l}31.0 \\
32.5 \\
33.3 \\
35.1\end{array}$ & $\begin{array}{l}148.0 \\
162.0 \\
159.0 \\
182.0\end{array}$ & $\begin{array}{l}35.6 \\
34.0 \\
39.1 \\
36.7\end{array}$ & $\begin{array}{r}7.5 \\
9.6 \\
10.0 \\
11.3\end{array}$ & $\begin{array}{l}267.0 \\
326.0 \\
391.0 \\
413.0\end{array}$ & $\begin{array}{l}6.0^{*} \\
6.6 \\
4.7 \\
4.0\end{array}$ & $\begin{array}{r}8.2 \\
9.6 \\
7.9 \\
10.0\end{array}$ & $\begin{array}{l}49.0 \\
63.0 \\
37.0 \\
40.0\end{array}$ \\
\hline & & & 163 & & & 349 & & & 47 \\
\hline $\mathrm{P}$ & $\begin{array}{l}6.0 \\
6.6 \\
6.3 \\
5.5\end{array}$ & $\begin{array}{l}23.6 \\
30.7 \\
28.6 \\
31.5\end{array}$ & $\begin{array}{r}142.0 \\
202.0 \\
181.0 \\
175.0 \\
175\end{array}$ & $\begin{array}{l}43.8 \\
44.2 \\
32.1 \\
41.6\end{array}$ & $\begin{array}{r}10.0 \\
9.3 \\
15.0 \\
8.6\end{array}$ & $\begin{array}{r}439.0 \\
402.0 \\
481.0 \\
357.0 \\
420\end{array}$ & $\begin{array}{l}4.3^{*} \\
8.6 \\
5.8 \\
6.1\end{array}$ & $\begin{array}{l}6.4 \\
7.2 \\
6.4 \\
5.7\end{array}$ & $\begin{array}{r}28.0 \\
63.0 \\
37.0 \\
35.0 \\
41\end{array}$ \\
\hline$Q$ & $\begin{array}{l}5.1 \\
6.0 \\
5.6 \\
5.9\end{array}$ & $\begin{array}{l}27.9 \\
29.6 \\
29.4 \\
32.8\end{array}$ & $\begin{array}{r}141.0 \\
177.0 \\
164.0 \\
195.0 \\
169\end{array}$ & $\begin{array}{l}33.7 \\
30.1 \\
33.8 \\
30.8\end{array}$ & $\begin{array}{r}11.1 \\
15.7 \\
9.9 \\
12.9\end{array}$ & $\begin{array}{r}373.0 \\
407.0 \\
334.0 \\
297.0 \\
378\end{array}$ & $\begin{array}{l}5.8^{E} \\
6.3 \\
4.5 \\
5.0\end{array}$ & $\begin{array}{r}8.7 \\
12.7 \\
7.8 \\
12.6\end{array}$ & $\begin{array}{r}51.0 \\
80.0 \\
35.0 \\
62.0 \\
57\end{array}$ \\
\hline$S$ & $\begin{array}{l}3.8 \\
3.9 \\
4.2 \\
4.3\end{array}$ & $\begin{array}{l}29.4 \\
25.6 \\
26.7 \\
29.4\end{array}$ & $\begin{array}{r}111.0 \\
100.0 \\
113.0 \\
125.0 \\
112\end{array}$ & $\begin{array}{l}21.6 \\
27.3 \\
56.6 \\
22.5\end{array}$ & $\begin{array}{r}17.8 \\
9.4 \\
10.0 \\
21.7\end{array}$ & $\begin{array}{r}384.0 \\
257.0 \\
566.0 \\
488.0 \\
424\end{array}$ & $\begin{array}{l}2.8^{\star} \\
2.7 \\
3.2 \\
1.8\end{array}$ & $\begin{array}{l}6.7 \\
4.4 \\
5.6 \\
6.1\end{array}$ & $\begin{array}{r}19.0 \\
12.0 \\
18.0 \\
11.0 \\
15\end{array}$ \\
\hline Mean & & & 152 & & & 520 & & & 31 \\
\hline
\end{tabular}


To evaluate the influence of the conditioning procedure (24 hours at $60{ }^{\circ} \mathrm{C}$ followed by conditioning to equilibrium at $23^{\circ} \mathrm{C}$ and 50 percent relative humidity) on the flame-spread index, four laboratories performed tests on polyurethane foam B specimens with and without the $60{ }^{\circ} \mathrm{C}$ preheating. These results, including means and standard deviations, are given in table 3.

One of the purposes of the interlaboratory evaluation of the test method was to determine the degree of uncertainty of the resultant data. Table 4 summarizes the results on $F, Q$, and $I$ in terms of the means, ranges, and the withinlaboratory and between-laboratory components of the coefficients of variation for each material (see appendix C). Coefficient of variation is the ratio of the calculated standard deviation to the mean, expressed as a percent. The within-lab and between-lab data are given in terms of the expected precision of a single run within a laboratory and a single run among laboratories, respectively. The values were derived from the analysis made separately for each material using all the reported data from the 13 laboratories. Because of the large discrepancy in the polyurethane result between Lab J and other labs, two sets of coefficients of variation, including as well as excluding the Lab J urethane data, were calculated. Appendix C defines the statistics for calculating the data in table 4 and is based on reference [5].

In general, repeatability (within-lab) would improve somewhat if it were based on the mean of four replicate tests. For a method of converting the statistical result based on a single test to that based on the mean of $n$ tests, also see appendix $\mathrm{C}$.

Figures 2 to 7 show typical data sheets including results for the three materials as reported by 3 labs. They also show procedures for calculating $F$ and $Q$, and for extrapolating $F$ from a single time-distance point observed in the neopreñe specimens.

\section{DISCUSSION}

\subsection{Variability and Source of Errors}

The $\beta$ values among laboratories are shown in table 1. The highest $\beta$ was reported by Lab $F$ and the lowest by Lab J and $K$. The effect of $\beta$ on the test results for Labs $F, J$, and $K$ is illustrated in figure 8 where the error for heat evolution, $Q$ appears to be systematic and directly related to the $1 / \beta$ value. In this figure, the average $Q$ of 4 replicate 


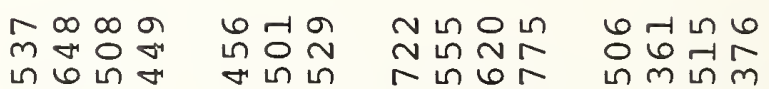

$+1$

$\infty$
$\infty$

$N$
$\infty$

$\oplus$
6

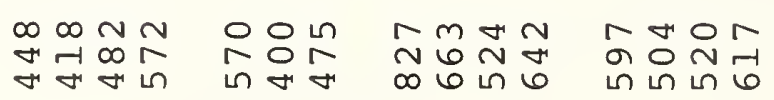

[4

$\mapsto$

$\mapsto$

$z$ 


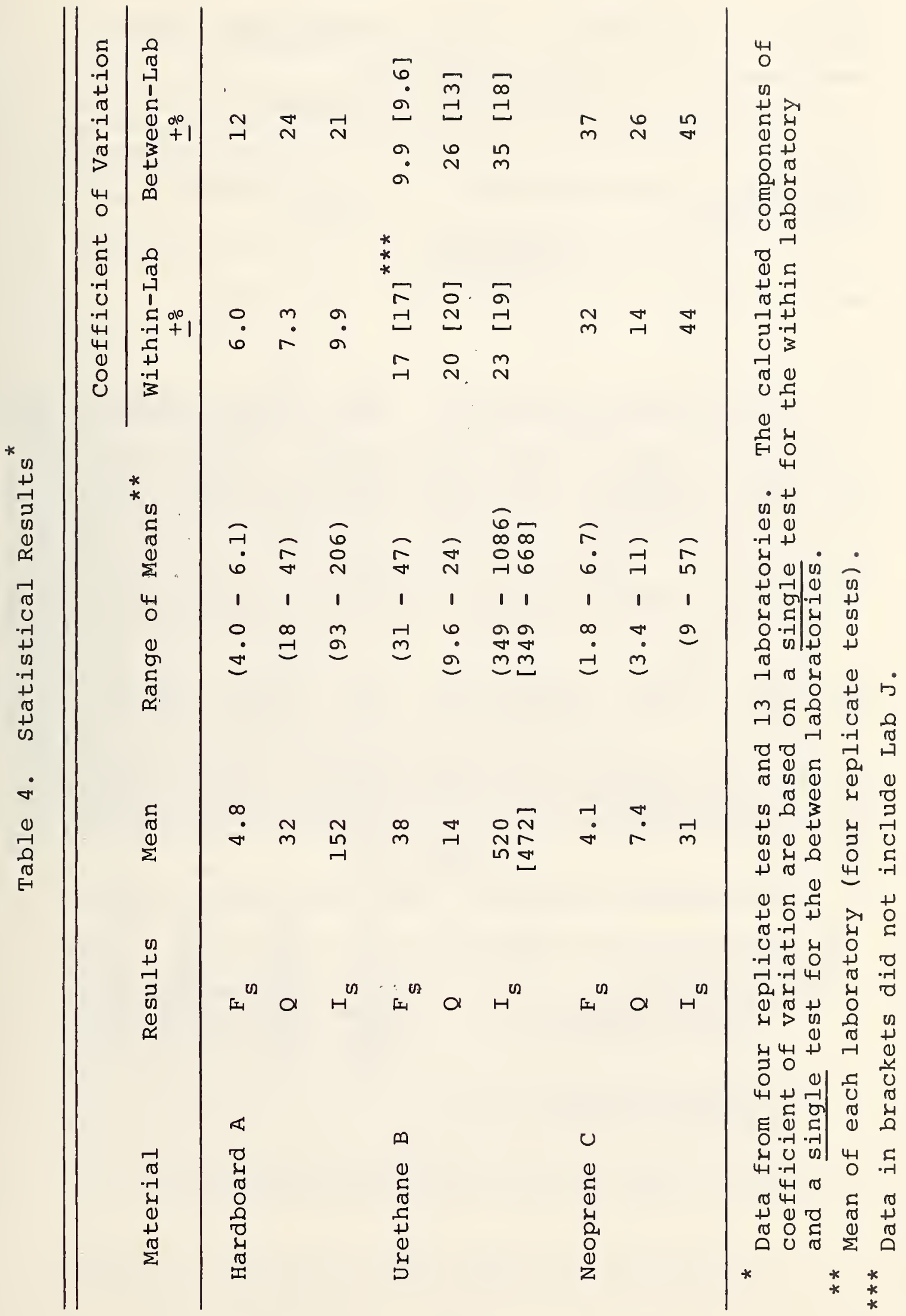


Sample Code No. $14-74-123-194$ Teat No. $\frac{2-6-7-9}{2}$ Date $12-3-7-4$ Teat by Aretoted by R.LAMIRAMLEE TIme

Sample Description: HARDERARD(NBS)

Manufacturer

Thlckness

Spectal Conditions
10. Dens1ty

1a. Denstey
$1 b / f t^{3}, 1 b / f t^{2}$ Moleture or $o z / \mathrm{gd}^{2}$

(A) Flame Spread Factor, $P_{8}=1+\frac{i}{T_{3}}+\frac{1}{T_{6}-T_{3}}+\frac{1}{T_{9}-T_{6}}+\frac{1}{T_{12}-T_{9}}+\frac{1}{T_{15}-T_{12}}$

(1)

\begin{tabular}{|c|c|c|c|}
\hline $\begin{array}{l}\text { D1e. } \\
\text { In. }\end{array}$ & $\begin{array}{l}\text { Mine } \\
\text { Min. }\end{array}$ & $\begin{array}{c}\text { Mae } \\
\text { Difference }\end{array}$ & $\begin{array}{l}\text { Reciprocal } \\
\text { Difference }\end{array}$ \\
\hline 3 & $1 . \bar{L} 4$ & 1.24 & .260 \\
\hline 6 & $19 \%$ & $: 70$ & 1.429 \\
\hline 9 & 3.16 & 1.22 & $\ldots 12 C$ \\
\hline 12 & 5.37 & 2.22 & $1 / 90$ \\
\hline-15 & 9.07 & 3.71 & .270 \\
\hline
\end{tabular}

MET

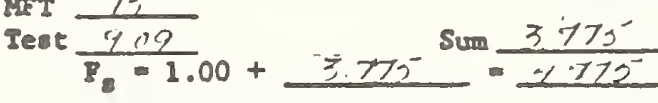

(3)

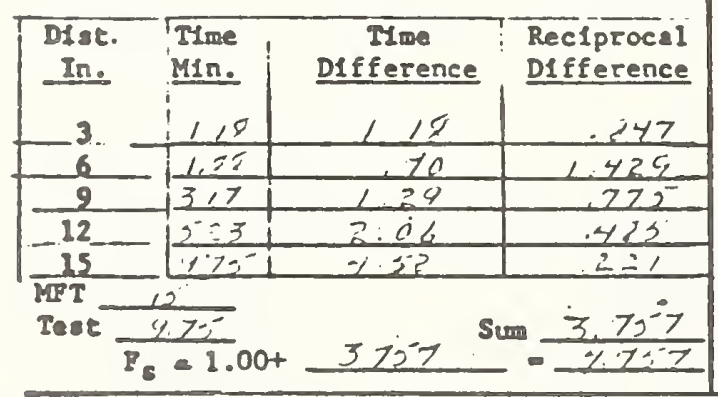

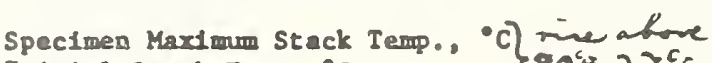
Intela l stack Temp. $\left.{ }^{\circ} \mathrm{C}\right\} 8 a^{\mathrm{c}} \mathrm{x} ; 7^{\mathrm{C}} \mathrm{C}$ Temp. Rise from specimen, ${ }^{\circ} \mathrm{C}$ Aobestos-Cement Board Stack Temp. Bise, 'C T, Net Stack Temp Rise, $C$ $Q=0.1 / B$ I, Heat Eva. Factor,

\footnotetext{
Note: MFT-Max, flame travel dutance, In. Test=Time at MF, wla.
}

Figure 2. Data Sheet of Lab O for Hardboard A

\begin{tabular}{|c|c|c|c|}
\hline $\begin{array}{l}\text { Dist. } \\
\text { In. }\end{array}$ & $\begin{array}{l}\text { Time } \\
\text { Min. }\end{array}$ & $\begin{array}{c}\text { Time } \\
\text { Difference }\end{array}$ & $\begin{array}{l}\text { Reclprocal } \\
\text { Difference }\end{array}$ \\
\hline 3. & 1.20 & 120 & .833 \\
\hline 6. & $\angle 27$ & .47 & 1.493 \\
\hline 9 & 3.12 & 1.12 & $.0 / r$ \\
\hline 12 & 4.97 & 1.75 & .513 \\
\hline 15 & 3.51 & $3 \times 1$ & $. i / c$ \\
\hline
\end{tabular}

(2)
(4)

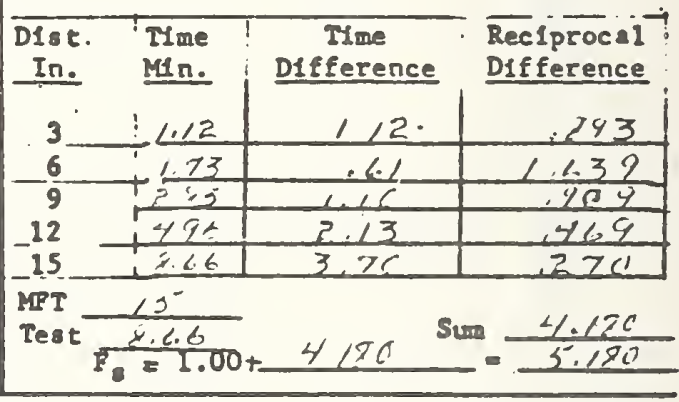

(1) $\frac{\frac{414}{171}}{\frac{-136}{\frac{141}{325}}}$ (2) (3) $\frac{\frac{-126}{172}}{\frac{244}{242}} \frac{124}{2391}$ (4)

$\frac{\frac{472}{1.79}}{\frac{25.3}{152}} \frac{2.16}{32.33}$
$445^{-}$ $\frac{174}{264}$ 112 $\frac{223}{514}$ 
RADIANT PANEL MEASUREMENTS - II

$$
\text { hab.o }
$$

(c) Flame Spread Index, $I_{s}=F_{s} \times Q$

(D) Plots of TIme vs Plame Travel

\begin{tabular}{|c|c|}
\hline 1) $I_{s}=4.773 \times 36.97=$ & $14 y$ \\
\hline 2) $I_{8}=4919 \times 32.50=$ & 162 \\
\hline 3) $I_{g}=4751 \times 33.33=$ & 159 \\
\hline 4) $I_{B}=5.18 C^{\prime} \times 3514$ & 182 \\
\hline & 163 \\
\hline
\end{tabular}

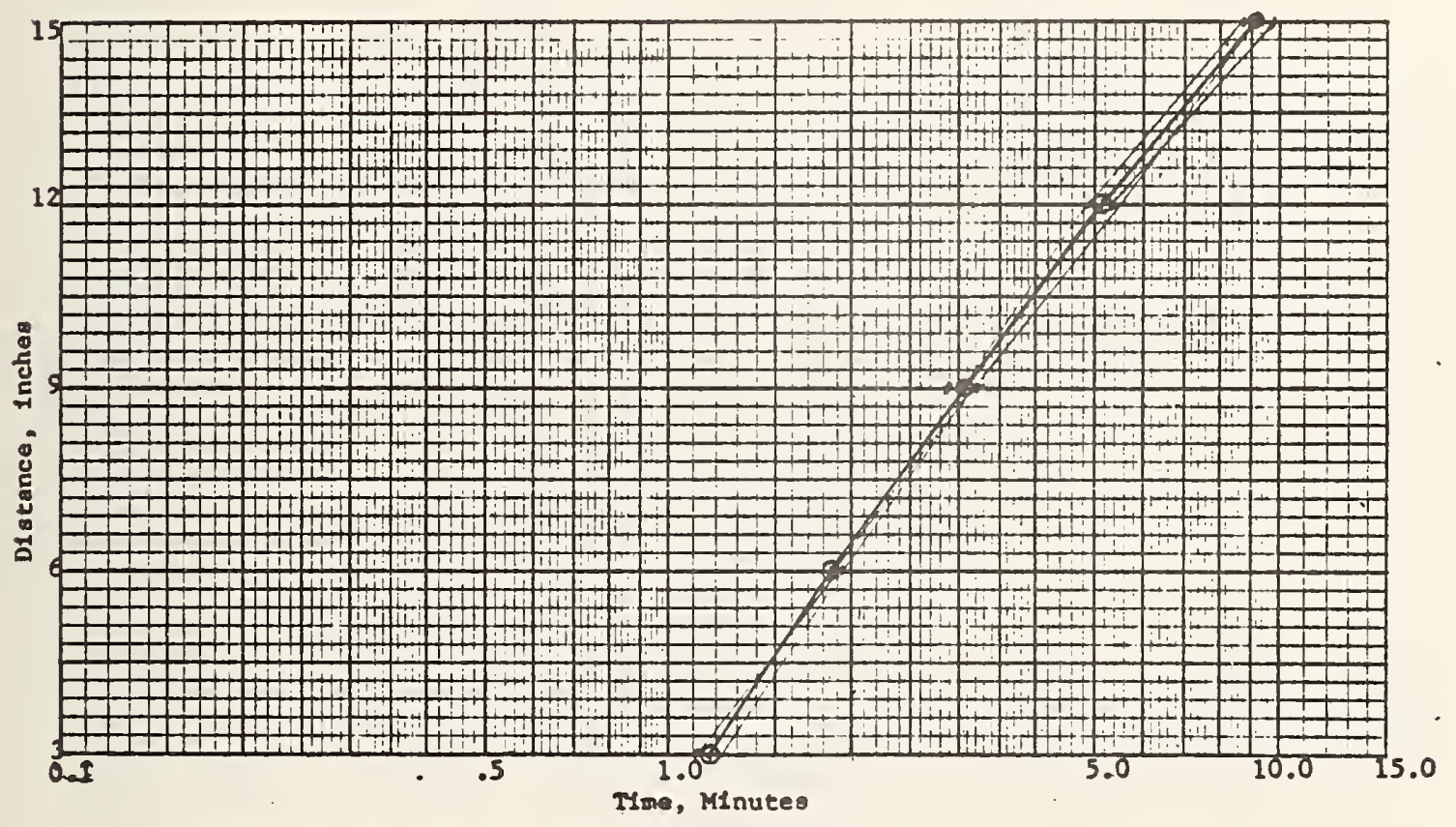

(E) Comments on Naturs of Burning, Melting, Flash Potential, etc., during test

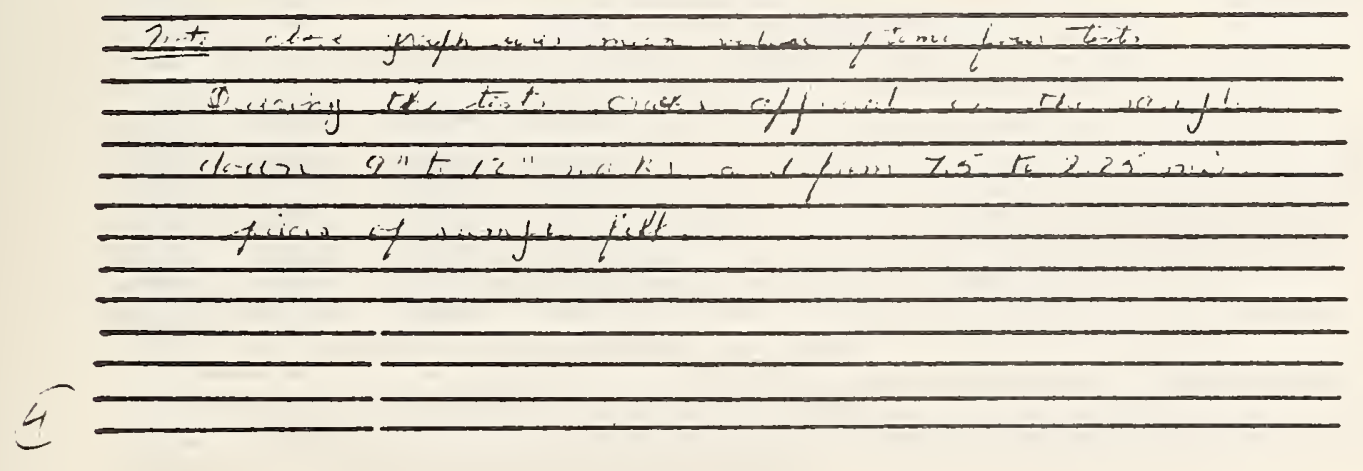

Figure 3. Plots of Flame Fronts of Hardboard A by Lab 0 
Sample Code No. $150,209,90,6$ Test No. $4,5,8,10$ Date $02-14-74$

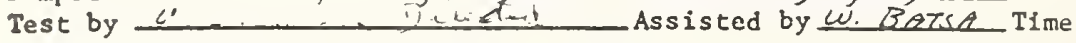

Sample Description: Neppeene Latex Foan

Manufacturer SuppLieo BY NATIDNCC BureaU of STANDARDS Thickness $/ 0$ in. Density $\mu \nu$. $1 \mathrm{~b} / \mathrm{ft}^{3}, 1 \mathrm{~b} / \mathrm{ft}^{2}$ Moisture $\%$

Special Conditions $60^{\circ} \mathrm{C} / 21 \mathrm{wr}_{\mathrm{H}} @ 23+3^{\circ} \mathrm{C}$ or $02 / \mathrm{yd}^{2} \pm 5 \% \mathrm{PH}$

(A) Flame Spread Factor, $F_{s}=1+\frac{i}{T_{3}}+\frac{1}{T_{6}-T_{3}}+\frac{1}{T_{9}-T_{6}}+\frac{1}{T_{12}-T_{9}}+\frac{1}{T_{15}-T_{12}}$

(1)

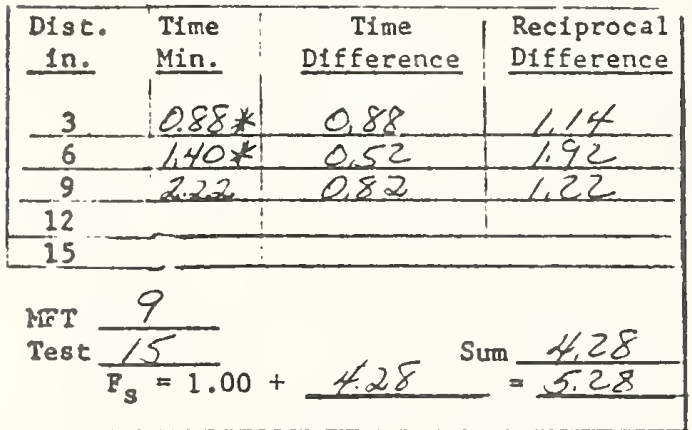

(3)

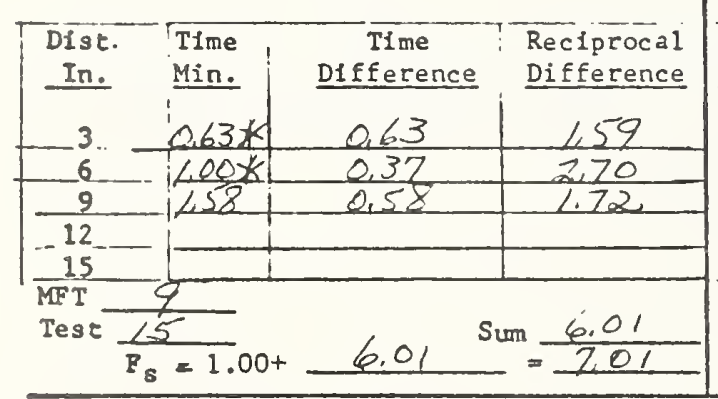

Specimen Maximum Stack l'emp., ${ }^{\circ} \mathrm{F}$

In1tial Stack Temp. 'F

Temp. Rise from specimen, ${ }^{\circ} \mathrm{F}$

Asbestos-Cement Board Stack Temp. Rise, ${ }^{\circ} \mathrm{F}$ $T$, Net Stack Temp Rise, ${ }^{\circ} \mathrm{F}$ $\mathrm{Q}=0.1 / \beta \mathrm{T}$, Heat Eva. Factor, $B=1.24$
(2)

\begin{tabular}{cc|c|c|} 
Dist. Time & $\begin{array}{c}\text { Time } \\
\text { In. }\end{array}$ & $\begin{array}{c}\text { Reciprocal } \\
\text { Din. }\end{array}$ & $\begin{array}{l}\text { Difference } \\
\text { Difference }\end{array}$ \\
\hline 3 & $0,57 *$ & 0,57 & 1,75 \\
\hline 6 & $0.90 *$ & 0,33 & 3,03 \\
\hline 9 & 143 & 0,53 & 1,89 \\
\hline 12 & & & \\
\hline 15 & & & \\
\hline
\end{tabular}

MET 9

Test $\frac{9}{\mathrm{~F}_{3}=1.00}+6.67$ sum $\frac{6.67}{7.67}$

(4)

\begin{tabular}{|c|c|c|c|}
\hline $\begin{array}{l}\text { Dist } \\
\text { In. }\end{array}$ & $\begin{array}{l}\text { Time } \\
\text { Min. }\end{array}$ & $\begin{array}{c}\text { Time } \\
\text { Difference }\end{array}$ & $\begin{array}{l}\text { Reciprocal } \\
\text { Difference }\end{array}$ \\
\hline 3 & $0.64 *$ & 0,64 & -156 \\
\hline 6 & $1,00 \%$ & 0.36 & 2,78 \\
\hline 9 & 161 & 0.61 & 1.64 \\
\hline 12 & & & \\
\hline & & & \\
\hline
\end{tabular}

MFT 9 Test $\frac{15}{F_{s}=1.00}+5.98$ sum $=\frac{5.98}{6.98}$

\begin{tabular}{|c|c|c|c|}
\hline (1) & $\begin{array}{r}(2) \\
472\end{array}$ & (3) & (4) 3 \\
\hline 16 & 396 & 391 & 396 \\
\hline 80 & 76 & 73 & 67 \\
\hline 8 & 7 & 8 & 8 \\
\hline$\frac{72}{6.61}$ & $\frac{69}{6.33}$ & $\frac{65}{546}$ & $\frac{59}{5.41}$ \\
\hline
\end{tabular}

Note: MFT $=$ Max. flame travel distance, in.

Test=Time at MFT, min.
$\nVdash=\operatorname{Ce} k$ from $D=D_{0}+6.5 \% \leftarrow$

Figure 4. Data Sheet of Lab E for Neoprene C 


\section{RADIANT PANEL MEASUREMENTS - II}

(c) Flame Spread Index, $I_{s}=F_{s} \times Q$ Neapletce $C$

\section{$a a_{c}=$}

- (I) $I_{8}=5.3 \times \underline{6.6}=35$

(D) Plots of Time vs Flame Travel

(c) (2) $I_{s}=7.7 \times 6.3=49$

$x$ (3) $I_{8}=7,0 \times 6,0=42$

(2) (4) $I_{3}=7.0 \times 5.4=38$ Average $=41$

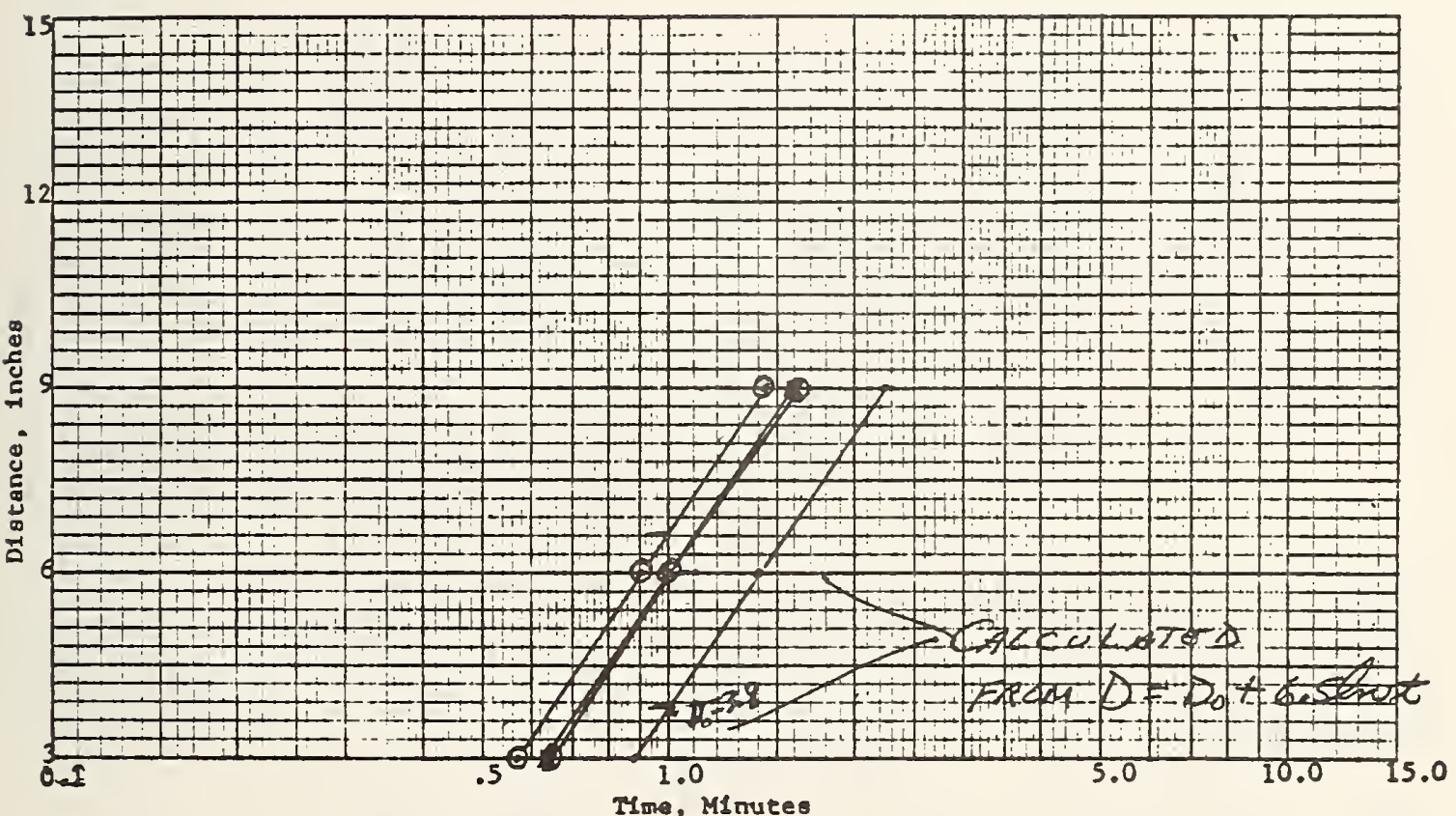

(E) Comments on Nature of Burning, Melelng, Flash Potentlal, etc., durlng test Seecimen began to flesta to g mart is son 25 chiter Echme contested Feanino

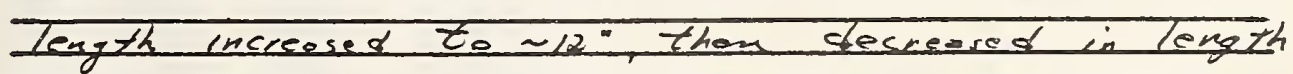
Until specimen lanted in losalejed spots whesh

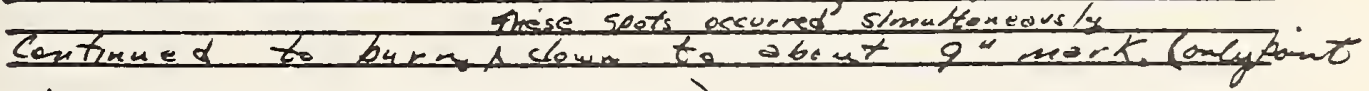

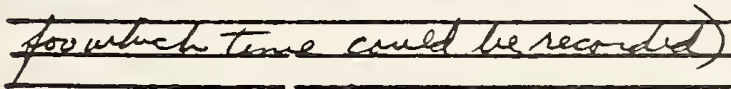

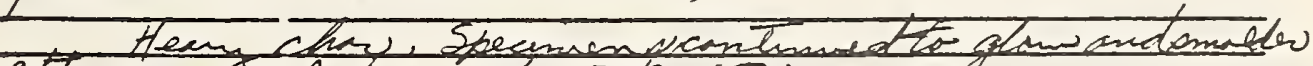

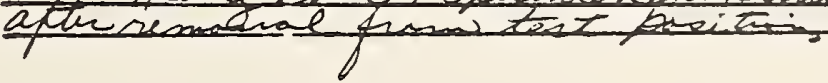

Figure 5. Plots of Flame Fronts of Neoprene C by Lab E 
Simple Code No.

Test by $\sqrt{\text { W SBANFOBD }}$

Test No.

fissisted by

Sample Description:-URELHane (W/AITE)

Manuf acturer

Thickness

1n. Density

$1 \mathrm{~b} / \mathrm{ft} \mathrm{t}^{3}, 1 \mathrm{~b} / \mathrm{Et}^{2}$ Molsture

$-\%$

Speclal Conditions or $0 z / \mathrm{yd}^{2}$

\section{RECORDED I:TTA}

(B) Flame Spread Factor, $F_{s}=1+\frac{i}{T_{3}}+\frac{i}{T_{6}-T_{3}}+\frac{1}{T_{9}-T_{6}}+\frac{1}{T_{12}-T_{9}}+\frac{1}{T_{15}-T_{12}}$

(1) 105

\begin{tabular}{|c|c|c|c}
\hline Dist. & Time & Time & Reciprocal \\
111. & Min. & Differcnce & DifEerence \\
\hline 3 & .15 & .15 & 6.67 \\
\hline 6 & .31 & .16 & 625 \\
\hline 9 & .49 & 18 & 5.56 \\
\hline 12 & .65 & .16 & 6.25 \\
\hline 15 & .84 & .19 & 526 \\
\hline
\end{tabular}

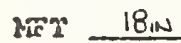

Test $\frac{=}{F_{s}=1.00}+29.99$ sum $=\frac{29.99}{30.99}$

(3) 177

\begin{tabular}{|c|c|c|c|}
\hline $\begin{array}{l}\text { Dist. } \\
\text { In. }\end{array}$ & $\begin{array}{l}\text { Time } \\
\text { Min. }\end{array}$ & $\begin{array}{c}\text { Time } \\
\text { Difference }\end{array}$ & $\begin{array}{l}\text { Reciprocal } \\
\text { Difference }\end{array}$ \\
\hline-3 & .14 & .14 & 7.14 \\
\hline 6 & .28 & .14 & 714 \\
\hline 9 & .38 & .10 & 15.00 \\
\hline$\ldots 12$ & .49 & .11 & 7.09 \\
\hline 15 & .01 & .17 & \\
\hline MFT & $18 \mathrm{iN}$ & \multirow{3}{*}{\multicolumn{2}{|c|}{ Sum $\frac{39.25}{-10.25}$}} \\
\hline Test & 1.19 & & \\
\hline & $F_{s}=1.0$ & & \\
\hline
\end{tabular}

Specimen Maximum Stack Temp., ${ }^{\circ} \mathrm{F}$

Initial Stack Terp. ${ }^{\circ} \mathrm{F}$

Temp. Rise from specimen, ${ }^{\circ} \mathrm{F}$

Asbestos-Cement Eoard Stack Te=p. Rise, ${ }^{\circ} \mathrm{F}$

$T$, Net Stack Temp Rise, ${ }^{\circ} F$

$Q=0.1 / \beta T$, Heat Eva. Factor,

$B=1.02$
(2) 9

\begin{tabular}{|c|c|c|c|}
\hline $\begin{array}{l}\text { Dist. } \\
\text { In. }\end{array}$ & $\begin{array}{l}\text { Time } \\
\text { Min. }\end{array}$ & $\begin{array}{c}\text { Time } \\
\text { Difference }\end{array}$ & $\begin{array}{l}\text { Reciprocal } \\
\text { Difference }\end{array}$ \\
\hline 3 & .15 & .15 & 6,67 \\
\hline 6 & .25 & .10 & 10.00 \\
\hline 9 & .30 & .13 & 7.69 \\
\hline 12 & .51 & 13 & 7.69 \\
\hline 15. & .72 & .21 & 4.76 \\
\hline
\end{tabular}

MET $18 \mathrm{~N}$

Test $\frac{1.20}{1.00}+\operatorname{sum} 36.81$

$P_{S}=1.00+30.81=\frac{36.81}{37.81}$

(4) 47

\begin{tabular}{|c|c|c|c|}
\hline $\begin{array}{l}\text { Dist. } \\
\text { In. }\end{array}$ & $\begin{array}{l}\text { Time } \\
\text { Min. }\end{array}$ & $\begin{array}{c}\text { Time } \\
\text { Difference }\end{array}$ & $\begin{array}{l}\text { Reciprocal } \\
\text { Difierence }\end{array}$ \\
\hline 3 &. .12 & .12 & -8.33 \\
\hline 6 & +.28 & .16 & 6.25 \\
\hline 9 & 41 & .13 & 769 \\
\hline 12 & 1.51 & .9 & 11.11 \\
\hline 15 & .68 & .18 & 5,56 \\
\hline
\end{tabular}

MFT IEIN.

Test Sum 3894 $F_{s}=1.00+38.94=39.44$
(1)

$\frac{\frac{580}{395}}{\frac{185}{35}} \frac{150}{\frac{150}{14.71}}$

(2)

$\frac{\frac{565}{395}}{\frac{170}{35}} \frac{\frac{132}{13,24}}{13}$

(3) $545-565$ $390-390$ $155-\frac{175}{40}$ $40-\frac{17}{435}$ $\frac{115}{11.27}-\frac{135}{13.24}$

Note: $M T T=H a x$, flame travel distance, in.

Test=Time at MHT, min.

Figure 6. Data sheet of Lab I for Polyurethane B 
URETHANE (WHITE)

RADIANT PANEL MEASUREMENTS - II

Leb. I

(c) Flame Spread Index, $I_{s}=F_{s} \times Q$

(D) Plots of Time vs Flame Travel

(1) $I_{s}=30.99 \times 14.71=\frac{455.9}{500.6}$
(2) $I_{s}=\frac{37.81}{453.24}=\frac{45.6}{528.8}$
(3) $I_{s}=\frac{40.25}{484.7}$
(4) $I_{s}=\frac{39.94}{\text { Average }}=\frac{13.24}{}=\frac{\frac{39}{11.27}}{}$

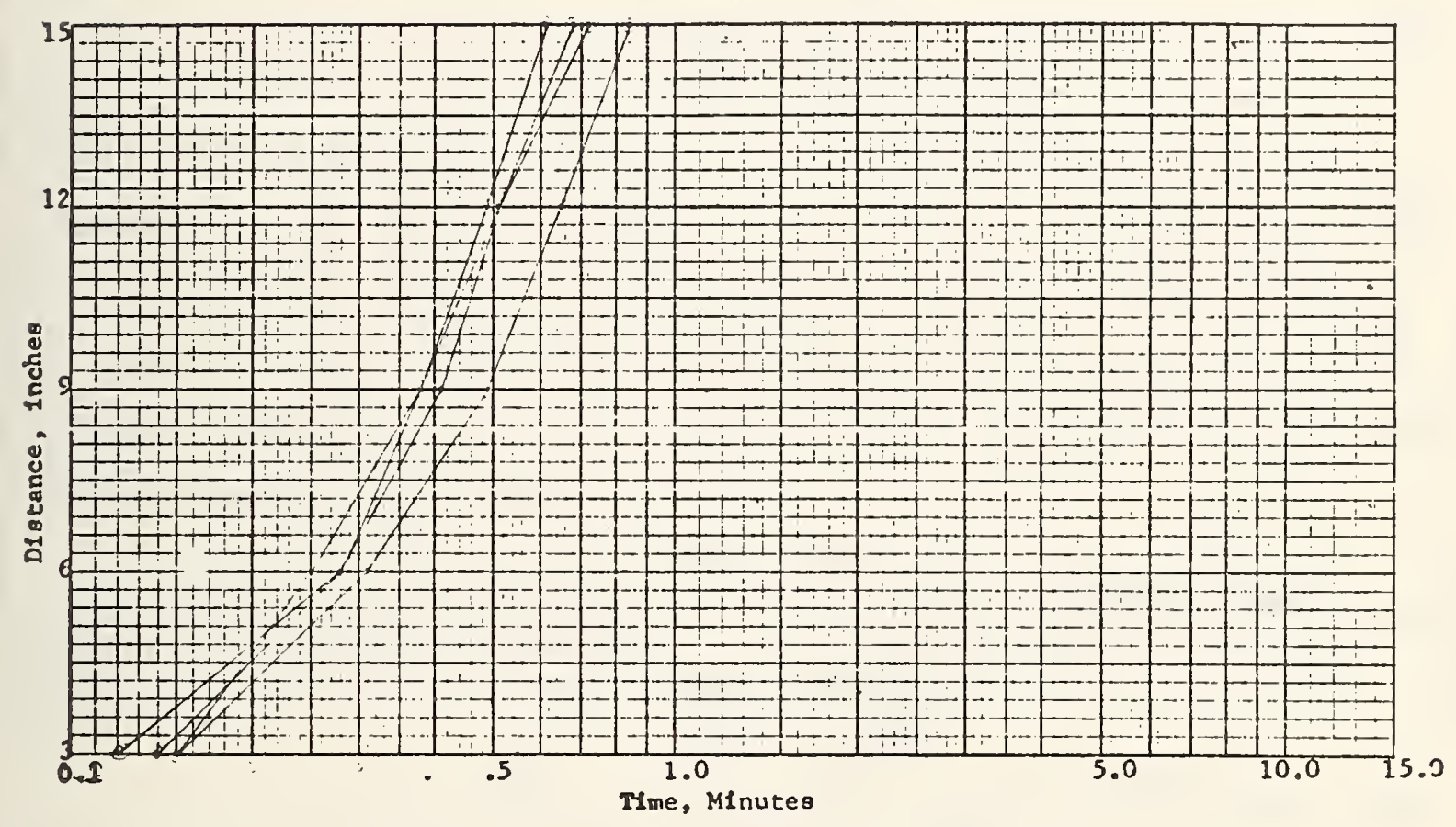

(E) Comments on Nature of Burning, Melting, Flash Potential, etc, during test Severe Drimage While Mectinis

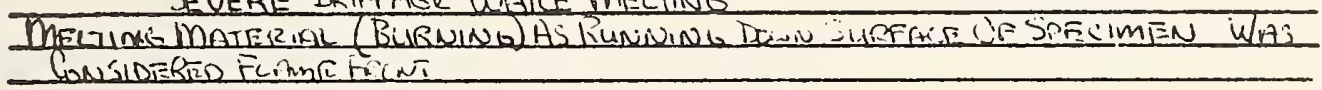

Figure 7. Plots of Flame Front of Polyurethane B by Lab I 


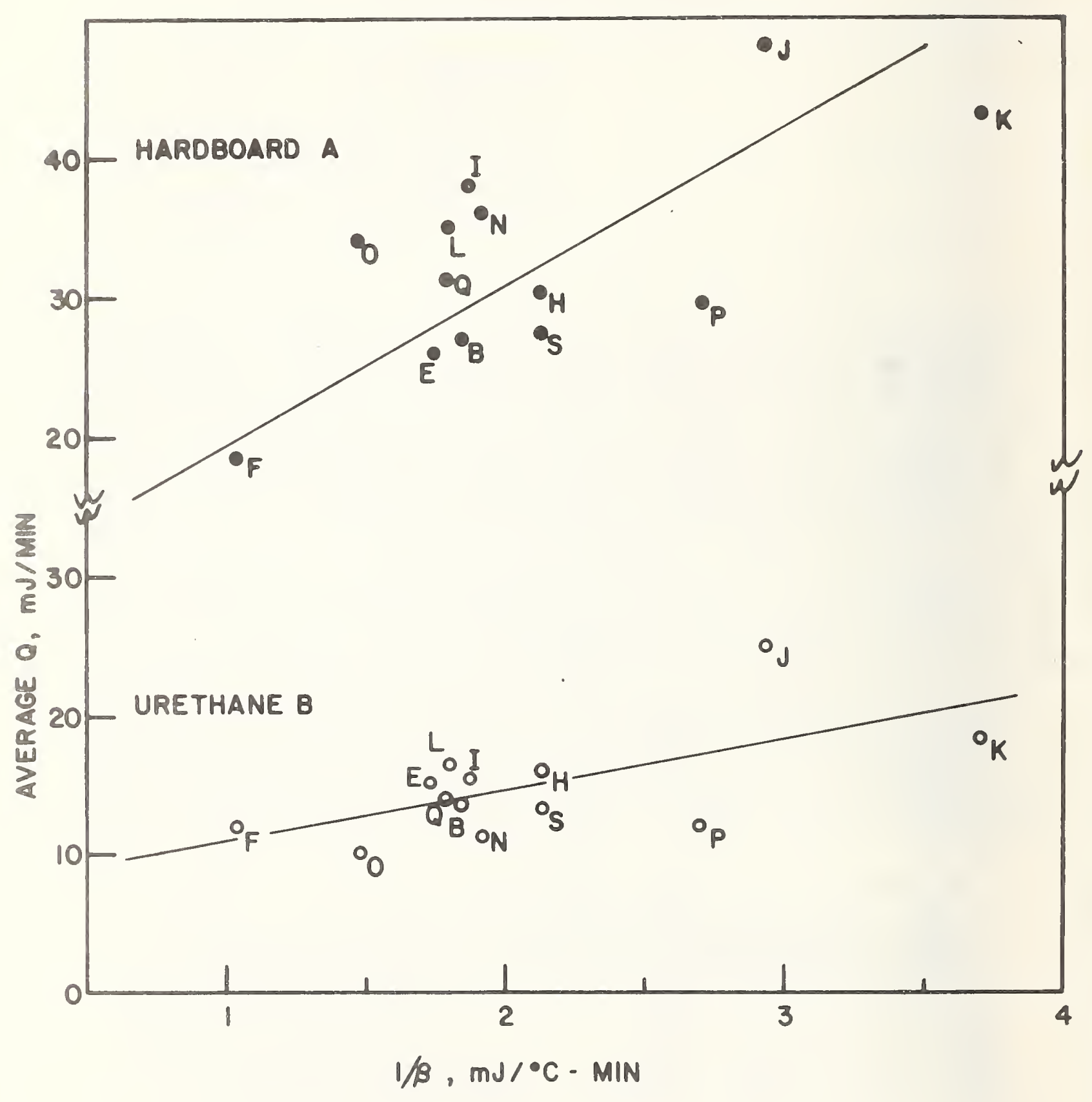

Figure 8. Correlation Between $Q$ and $1 / B$ for All Labs 
runs are plotted against the $1 / \beta$ where the curves are based on the least square fit of the data. The large deviation of $Q$, at least for labs $F, J$ and $K$, was affected by error in determining $\beta$. This seems to indicate that calibration of $\beta$ may be the most critical source of variability in calculating the flame-spread index among labs.

The pyrometer to panel distances shown in table 1 appear to center around $1.27 \mathrm{~m}$ except for labs $\mathrm{J}(0.81 \mathrm{~m}), \mathrm{S}(0.91 \mathrm{~m})$ and $0(2.49 \mathrm{~m})$. Since the distance required for viewing depends on the type of lens and the size and location of the aperture, distances reported by the three labs might be correct for their type of pyrometers.

The range of the maximum temperature increases $\left(\Delta \mathrm{T}_{\mathrm{m}}\right)$ among the laboratories for the three materials and the corrections for the temperature rise $\left(\Delta T_{C}\right)$ of the asbestoscement board ( $A C B)$ specimen are also shown in table 1 . The temperature correction is based on the time-temperature response of the asbestos-cement board blank. The correction to be applied should correspond to the time at which the maximum stack thermocouple temperature was observed during each test. Five laboratories used the same correction value throughout, even though the times to reach the maximum temperature were different for the different specimens. The ratio of $\Delta T_{C}$ to $\Delta T_{m}$ for Hardboard $A$, for example, varied from 0 to $13 \%$ among the labs.

To minimize this source of error, an average timetemperature rise curve should be drawn based on many tests using the ACB blank specimens. The appropriate temperature correction should then be selected from the curve to suit each test.

Results on the SRM $1002 \mathrm{~b}$ material appear reasonably consistent among all of the reporting labs except for Lab $\mathrm{P}$, which has the highest $F_{S}(7.3)$ and lowest Q (27.1). High $F_{s}$ to $Q$ ratios were also exhibited in the other three materials tested by Lab P. The reasons for this are not known at present.

Data from the statistical analysis in table 4 show that within-lab variability of both $F_{s}$ and $Q$ for Hardboard $A$ were relatively low and comparable. On the other hand, the component of between-lab variability for both $F_{S}$ and $Q$ were much higher, particularly the coefficient of variation for Q. These results are probably typical of materials with relatively uniform burning characteristics. For the cellular plastic materials, both the within-lab and between-lab coefficients of variation were considerably larger. 
The largest source of variability was associated with Neoprene $\mathrm{C}$. The burning characteristic of this specimen was such that flames repeatedly flashed down from the top of the specimen during the early part of the test exposure. Since the duration of the flash was less than 3 seconds it was not considered a sustained flame front in accordance with the method. After an exposure of 1 to 3 minutes, small cracks began to develop between the 6 and 9 inch levels thus creating anchoring locations for the flame. Under these conditions, a stable flame usually developed at one of the crack openings. This time was recorded. Since this phenomenon was variable, large within- and between-lab differences for $\mathrm{F}_{\mathrm{S}}$ of Neoprene $\mathrm{C}$ resulted.

Paragraph 8.5 of the test method standard describes a method of calculating a flame-spread factor, $F_{S}$, for cases such as the one mentioned above where flames skipped one or two distance increments and anchored in one position without further spread. By using the prescribed equation from the standard, $D_{t}=D_{O}+6.5$ ln $t$, where the distance $\left(D_{t}\right)$ is in inches and the corresponding time $(t)$ is in minutes, $D_{O}$ corresponding to a time of 1.0 minutes can be calculated. Based on this derived $\mathrm{D}_{\mathrm{O}}$, other corresponding times for distances of $3,6,9$ or 12 inches and $F_{S}$ may be determined by the above method.

Fs data on Neoprene $\mathrm{C}$ in table 2 identified by an "E" were from the 5 labs using this method. Data identified by an asterisk were derived by the author using the above equation from the reported single time-distance values from 4 labs. The data from the remaining 3 labs indicated that 2 or more time-distance increments were indeed observed in each test and therefore required only normal treatment.

\subsection{Effect of Preheating Urethane}

Table 3 shows that the flame-spread index values for Urethane $B$ were not significantly affected by preheating of the specimens $\left(60^{\circ} \mathrm{C}\right.$ for 24 hours) prior to conditioning.

\subsection{Rank Ordering of Laboratories}

Rank ordering of laboratories in terms of aggregate flame-spread index value shows that Labs $\mathrm{J}$ and $\mathrm{K}$ ranked among the three highest laboratories for both Hardboard A and Urethane B, whereas Lab F ranked the lowest for Hardboard A and Neoprene $\mathrm{C}$. These three laboratories also reported values with unusually high deviations relative to those of others. Comparison for all the labs are illustrated in 
figure 9 where the mean of all laboratories for a material is plotted against the mean (four replicates) of each laboratory in log-log coordinate. The graph shows the need for labs with consistantly high or low values to examine their procedures [7].

\subsection{Effect of Procedure Modifications}

The modifications adopted by the participants in this study were found to be satisfactory. No pilot "flameout" was reported in any test using the new burner. The aluminum back and side wrapping and front screening alleviate the dripping problem. Cleaning of thermocouples after each run was considered rather difficult even with the use of the compressed air jet. The newer commercial model of the apparatus uses a retrievable thermocouple bank which can easily be withdrawn from the stack for cleaning after each test.

\subsection{Analysis of Data}

The interlaboratory variability ranged from 21\% for Hardboard A to 45\% for Neoprene C in terms of coefficient of variation for flame-spread index. The higher variability for Neoprene $C$ was due in part to the difficulty of defining the flame front and the problem of a low stack temperature rise, which was strongly affected by even a small error in the base temperature correction for the ACB board. It also had a relatively low flame-spread index (mean $I_{s}=31$ ), for which a higher coefficient of variation may be expected.

Table 2 shows that the magnitude of $I_{s}$ for Polyurethane $B$ as measured by Lab J was about twice that of the average for all the other labs. If the urethane data of Lab $\mathrm{J}$ were excluded in the analysis, the between-laboratory component of coefficient of variation could be reduced from 26 and 35 percent to only 13 and 18 percent for $Q$ and $I_{s}$, respectively, as shown in table 4 .

The within-lab coefficient of variation of $I_{s}$ obtained by 13 labs for hardboard was 9.9\%. This was only somewhat higher than the value of $5.9 \%$ (12 runs) determined by a single lab, NBS, for the standard reference material 1002b, which was also a hardboard material having an $I_{s}$ value of 190 [6].

The generally higher variability of $Q$ in the betweenlab coefficient of variations for all three materials indicates the need for better control of the parameters that affect $Q$ among the labs. This involves primarily the calibration constant $\beta$. To pinpoint the source of error from 


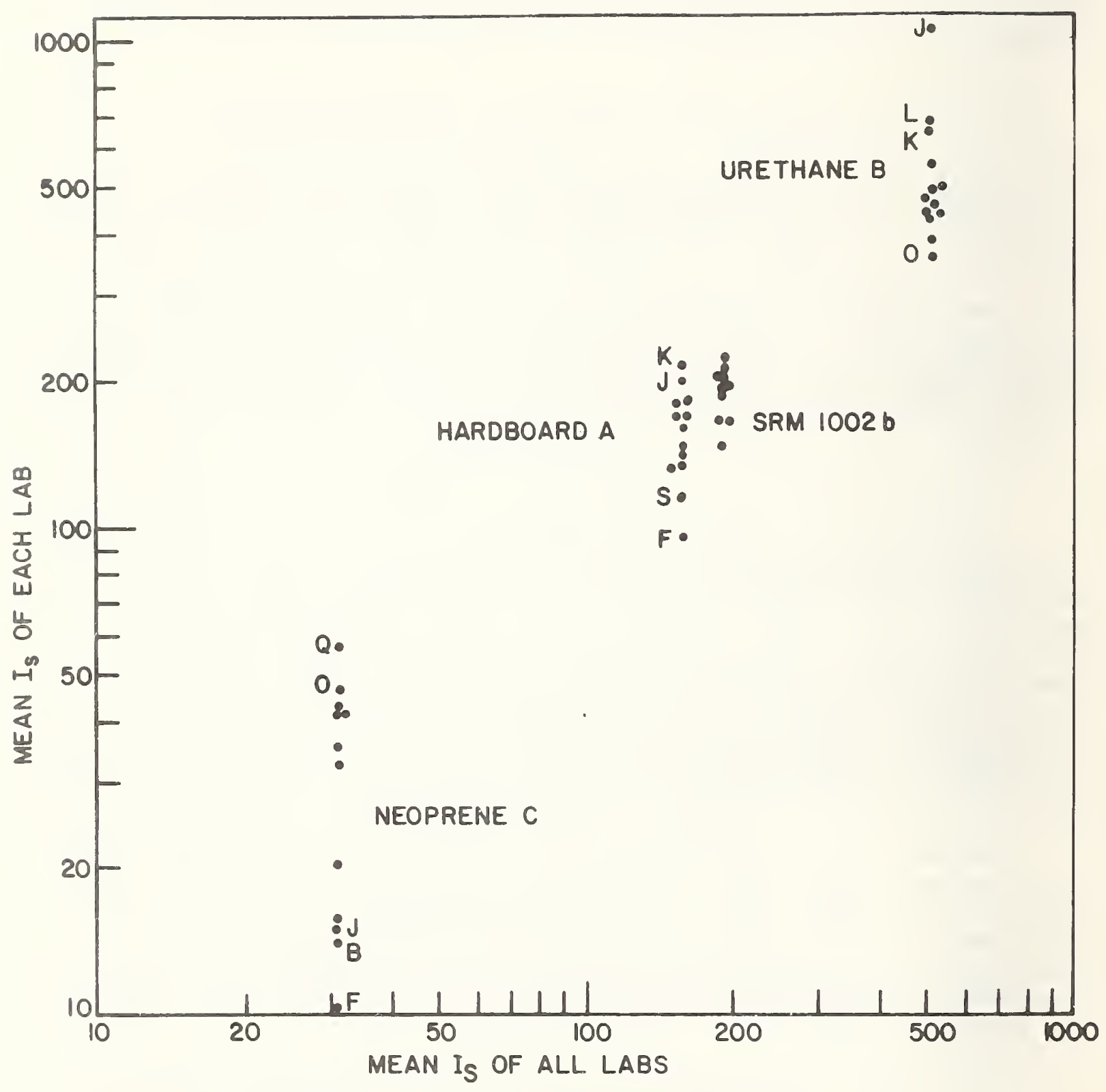

MEAN Is OF EACH LAB. VERSUS MEAN IS FOR ALL LABS

Figure 9. Mean $I_{S}$ of Each Lab Versus Mean $I_{s}$ for All Labs 
$\beta$, more information would be required on the gas flow measuring devices and other details of the calibration procedure used by each lab. But to minimize this type of error in the future, a more detailed description on instruments and procedures for determining $\beta$ should be given in the method. Continuous soot deposits on thermocouples during a test also play a role in the measurement precision of Q. However, this is difficult to correct. Automatic cleaning of the thermocouple bank after each run should be required.

Though this method was particularly useful for tests of materials with steady and definite flame fronts, the current study shows that the method can also be used for slow-melting materials with less stable flame fronts. However, higher coefficients of variation in test results are to be expected because of the additional uncertainty in the $\mathrm{F}_{\mathrm{S}}$ introduced by the slight melting and occasional flashing experienced by these elastomeric materials.

\section{SUMMARY AND CONCLUSIONS}

Based on the reported test results of 4 replicate runs for each of 3 test materials, one reference material, and the calibration values on $\beta$ from 13 participating laboratories in the radiant panel study, the following conclusions are justified:

1. The within-laboratory coefficient of variation of the flame-spread index, Is for Neoprene $C$ and Urethane $B$ were about 4 times and 2 times higher than the Hardboard A specimens, respectively. The higher variability for the flexible foams may reasonably be attributed in part to the difficulty of reading the flame front of a melting Urethane $B$ and the unstable flame front of Neoprene $C$ specimens. The base temperature correction errors on the low stack temperature rise from the Neoprene $C$ specimens also affected the variability.

2. The between-laboratory component of coefficient of variation of $I_{S}$ ranged from \pm 21 \% for hardboard to $\pm 45 \%$ for neoprene.

3. The correlation between the magnitude of the $Q$ and the $\beta$ values for some labs indicate that the betweenlaboratory variation can be reduced by the use of a more explicit procedure and technique in determining the $\beta$ values during calibration by each laboratory. 
4. The adopted modifications such as the new pilot burner, aluminum wrapping, and screen cover in the test procedures for melting foam specimens, alleviated much of the difficulty of testing this type of specimen.

5. The flame-spread index, $I_{S}$, for Urethane $B$ was not significantly affected by preheating the specimens to $60{ }^{\circ} \mathrm{C}$ for 24 hours prior to conditioning of the specimen at $23^{\circ} \mathrm{C}$ and $50 \%$ relative humidity.

6. In the absence of a more suitable test for flame spread of materials that melt and drip, this method with the stated modifications appears to be suitable for the type of elastomeric materials represented by the Neoprene $\mathrm{C}$ and the slow-melting Urethane B specimens. Because the test results have inherently higher coefficients of variation, additional work needs to be done to establish the type of behavior to be expected from these types of materials. Judgment should be used in the application of this test and in establishing criteria for regulatory purposes.

\section{ACKNOWLEDGMENTS}

The author wishes to thank the individuals and organizations listed on page 3, for their participation in performing the tests and making the data available. Their contributions in the discussions on the testing procedures are very much appreciated.

\section{REFERENCES}

[1] Standard Method of Test for Surface Flammability of Materials Using a Radiant Heat Energy Source, ASTM E 16267, Annual Book of ASTM Standards American Society for Testing and Materials, Philadelphia, 1973, Part 14.

[2] Robertson, A. F., Surface Flammability Measurements by the Radiant Panel Method, ASTM Special Technical Publication No. 344, American Society for Testing and Materials, 1962 .

[3] Gross, D. and Loftus, J. J., Surface Flame Propagation on Cellulosic Materials Exposed to Thermal Radiation, J. Res., Nat. Bur. Stand. (U.S.), $67 \mathrm{C} 3$ (Eng. and Instr.) 251-258 (1963).

[4] Gross, D., Private Communication (NBS Report 6319), 1958. 
[5] Mandel, John, Repeatability and Reproducibility, Matl. Res. and Stand. (ASTM) Vol. 11, No. 8 (1971).

[6] National Bureau of Standard SRM 1002b certificate.

[7] Youden, W. J., Graphical Diagnosis of Interlaboratory Test Results, Precision Measurement and CalibrationStatistical Concept and Procedures, Nat. Bur. Stand. (U.S.) Special Publication 300 (1969). 
APPENDIX A. DETAILS ON TEST APPARATUS

AND PROCEDURE MODIFICATIONS OF THE RADIANT PANEL METHOD

Radiant Panel:

1. Btu-rated gas or CP grade-methane should be used to determine the $\beta-v a l u e$ of the panel in the calibration prior to the round robin.

2. In determining the $\beta$-value, the higher (gross) heating value of the gas should be used. Corrections for vapor pressure (wet gas meter), and other measurement parameters from standard temperature and pressure conditions are required (see end of appendix for sample calculation).

3. Align and position the pyrometer properly. The proper position for the radiation pyrometer, when monitoring the output of the radiant panel, is along the normal through the center of the panel surface, and at a distance which would allow the pyrometer to view a central circular area 250-mm in diameter on the panel. This position should be determined as follows: With the panel operating and the pyrometer sighting along the normal, slowly insert an incombustible opaque shutter in front of the panel from the top, the bottom and each side, in turn. Note how far the shutter must be inserted before the.pyrometer output reading starts to decrease. Adjust the pyrometer along the normal until the position is found at which the output starts to decrease when the shutter is $25 \mathrm{~mm}$ from each side and $100 \mathrm{~mm}$ from the top or bottom of the panel. The pyrometer should be fixed in this position on a sturdy mount.

4. Calibrate the pyrometer if it has not been checked during the past 2 years. (NBS will provide this service.)

5. Modify pilot burner assembly and adjust the flow rates of gases to within $+3 \%$ of the suggested values of $0.9 \mathrm{l} / \mathrm{min}$ for acetylene and $4.5 \mathrm{l} / \mathrm{min}$ for air. Figure 1 shows the construction and location of the pilot burner which would resist "blow-out" by flame inhibitors from the test specimens.

6. A typical radiant panel maintained at a blackbody temperature of $670^{\circ} \mathrm{C}$ requires a gas flow rate of about $22 \mathrm{l} / \mathrm{min}$ for natural gas $\left(>95 \% \mathrm{CH}_{4}\right)$ and $316 \mathrm{\ell} / \mathrm{min}$ for air. 
7. Determine the stack temperature rise from the asbestos-cement board (ACB) with the new pilot burner as function of time. Monitor the equilibrium base line temperature of the stack for at least 4 minutes with the pilot flame on and in front of the radiant panel. Then insert the blank specimen consisting of a $6.4-\mathrm{mm}$ thick asbestoscement board and a 13-mm thick asbestos millboard backing in the sample holder. The temperature rise above the base line, as a function of time shall be used to determine the correction for the $A C B$ temperature rise. In normal test runs, the pilot flame should also be on and in front of the panel prior to sample insertion.

Specimen Preparation:

1. All specimens including the hardboard shall be heated at $60{ }^{\circ} \mathrm{C}$ for 24 hours and then conditioned one week (minimum) at $23+3{ }^{\circ} \mathrm{C}$ and $50+5 \%$ relative humidity.

2. Prepare aluminum foil (0.05 $\mathrm{mm}$ thick) wrapping frame to fit each specimen. Install the wrapper on the specimen before insertion into sample holder for testing. A solid mold, identical to the specimen in size, should be used to form the frame to avoid unnecessary wrinkles on the foil. Cut off excessive foil at the frame edges of the holder.

3. Mount poultry netting screen (25-mm hexagonal wire mesh, 0.8-mm diameter wire) in the specimen holder and flush against the exposed face of the specimen for all foam materials.

4. Use 3.2-mm thick asbestos-cement board as backing for the foam specimens and 13-mm thick asbestos millboard for the hardboard specimen.

5. Do not compress the flexible foam specimen while loading it into the holder.

Recording and Thermocouple Cleaning:

1. Record stack temperature by a continuous recorder or a multi-point recorder with 5-second or less interval per print channel. 
2. Flame front is defined as the maximum advance of flame lasting 3 seconds or more. Flaming streak associated with melted material moving down the middle of the specimen will be considered as a flame front. However, intermittent flashing is not considered as a flame front unless it has stabilized for more than 3 seconds. Timing of the flame front arrival at the 3 -inch interval lines on the specimen should be consistent. For example, if the arrival time is defined such that half of the length of the interval line is traversed by the visible flame front, the same definition should be used for all subsequent interval lines.

3. All thermocouples in the stack should be cleaned (free of soot deposits) prior to each test run.

A compressed air jet impinging on the thermocouple from small nozzles in a $25-\mathrm{mm}$ diameter tube inserted from a side of the stack was found to be effective in dispersing the soot.

Test Sequence and Data Sheet:

1. A code letter is assigned to each laboratory and labeled with data sheets.

2. Record the required information on the data sheets including a sample of stack temperature measurement on recorder paper; give data on $\beta$ calculations and plot time-distance data on the graph papers provided. 


\section{APPENDIX B. SAMPLE CALCULATIONS FOR HEAT INPUT RATE CORRECTION USED IN CALIBRATION OF RADIANT \\ PANEL STACK THERMOCOUPLES}

The following examples show how the required higher or gross heating value (HHV), which included the heat of condensation of $\mathrm{H}_{2} \mathrm{O}$ in the combustion product, may be corrected for the difference between volumes measured under your ambient conditions and under the standard conditions. Heating values for fuel gases are reported in terms of the standard temperature, $\mathrm{T}_{\mathrm{S}}=60^{\circ} \mathrm{F}^{*}$ and pressure, $\mathrm{P}_{\mathrm{S}}=30.0$ inch $\mathrm{Hg}$ and under water saturated basis as described by ASTM D 1071. Ta and $\mathrm{P}_{\mathrm{a}}$ are ambient temperature and pressure during the calibration, respectively.

Assume: HHV of the Btu-rated methane $=1000 \mathrm{Btu} / \mathrm{ft}^{3}(60$ ${ }^{\circ} \mathrm{F}, 30.0$ in $\mathrm{Hg}$, saturated).

(A) If the flow rate was measured by a wet gas meter at these ambient conditions:

Temperature (water)

Pressure drop (meter)

Atm. Pressure

Gas flow rate

Vapor Pressure

$$
\begin{aligned}
& \mathrm{P}_{\text {Wa }}=1.42^{\prime \prime} \mathrm{Hg}\left(90^{\circ} \mathrm{F}\right) \\
& \mathrm{P}_{\text {WS }}=0.52 " \mathrm{Hg}\left(60^{\circ} \mathrm{F}\right) \\
& \mathrm{T}_{\mathrm{a}}=90^{\circ} \mathrm{F} \quad \mathrm{T}_{\mathrm{S}}=60^{\circ} \mathrm{F} \\
& \Delta \mathrm{p}=1^{\prime \prime} \mathrm{H}_{2} \mathrm{O}=\frac{1}{13.6}=0.074 " \mathrm{Hg} \\
& \mathrm{P}_{\mathrm{a}}=30.15^{\prime \prime} \mathrm{Hg} \quad \mathrm{P}_{\mathrm{s}}=30.00 " \mathrm{Hg} \\
& \mathrm{V}=\mathrm{V} \mathrm{ft} \mathrm{t}^{3} / \mathrm{min}
\end{aligned}
$$$$
\text { Density of } \mathrm{Hg}=13.6
$$

Correction from standard conditions to measuring conditions:

$$
\begin{aligned}
\text { HHV } & =1000 \times \frac{459.7+T_{S}}{459.7+T_{a}} \times \frac{P_{a}+\Delta P-P_{w a}}{P_{S}-P_{\text {wS }}} \\
& =1000 \times \frac{459.7+60}{459.7+90} \times \frac{30.15+0.07-1.42}{30.00-0.52} \\
& =924 \mathrm{Btu} / \mathrm{ft}^{3}
\end{aligned}
$$

* To make metric conversion in Appendix B see the SI Conversion Chart in Appendix $\mathrm{Bl}$. 
(B) If flow rate was measured by a dry meter, $\left(\triangle \mathrm{P}=1 " \mathrm{H}_{2} \mathrm{O}\right)$ the corrected HHV will be:

$$
=1000 \times \frac{459.7+60}{459.7+90} \times \frac{30.15+0.07-0}{30.00-0.52}
$$

$\mathrm{HHV}=969 \mathrm{Btu} / \mathrm{ft}^{3}$

If the calibration gas is pure methane (99.93\%) the following heating values may be used:*

$997.4 \mathrm{Btu} / \mathrm{ft}^{3}, \mathrm{H}_{2} \mathrm{O}$ saturated, at $60^{\circ} \mathrm{F} 30.0 " \mathrm{Hg}$

(wet gas meter)

$1015.0 \mathrm{Btu} / \mathrm{ft}^{3}, \mathrm{dry}$, at $60^{\circ} \mathrm{F}, 30.0^{\prime \prime} \mathrm{Hg}$

(C) If the conditions during measurement using a rotameter (dry) were:

$$
\begin{aligned}
\mathrm{T}_{\mathrm{a}} & =80{ }^{\circ} \mathrm{F} \text { (gas temperature) } \\
\mathrm{P}_{\mathrm{a}} & =30.15^{\prime} \mathrm{Hg}, \Delta \mathrm{P}=0, \text { the correction will be: } \\
\mathrm{HHV} & =1015.0 \times \frac{459.7+60}{459.7+80} \times \frac{30.15}{30.0} \\
& =982 \mathrm{Btu} / \mathrm{ft}^{3}
\end{aligned}
$$

If a rotameter is used, it should be calibrated for methane and corrected for temperature. It should not be based on that of air. The pressure drops of the rotameter should be included in the calibration and no additional correction is necessary. If the rotameter is calibrated under other (temp., pressure) conditions, the appropriate corrections should be made.

\footnotetext{
Values from Eiseman and Potter, J. of Res., Natl. Bur. Stand., Vol. 58, p. 213 (1957).
} 


\section{APPENDIX BI. SI CONVERSION UNITS}

In view of the present accepted engineering practice in this country, common U.S. units of measurement have been used throughout this report. In recognition of the position of the United states as a signatory to the General Conference on Weights and Measurements which gave official status to the metric SI system of units in 1960, we assist the readers interested in making use of the coherent system of SI units by giving conversion factors applicable to the U.S. units used in this report.

Length

1 in $=0.0254$ meter

$1 \mathrm{ft}=0.3048$ meter

Volume

$1 \mathrm{ft} \mathrm{t}^{3}-0.0283 \mathrm{~m}^{3}$

Mass

$11 \mathrm{~b}-0.4536 \mathrm{kilogram}$

Density

$1 \mathrm{pcf}\left(1 \mathrm{~b} / \mathrm{ft}^{3}\right)=0.0160 \mathrm{~g} / \mathrm{cm}^{3}$

Temperature

Temperature in ${ }^{\circ} \mathrm{F}=9 / 5$ (temperature in ${ }^{\circ} \mathrm{C}$ ) $+32{ }^{\circ} \mathrm{F}$

Energy

1 Btu $=1054.6$ joules

Power

$1 \mathrm{Btu} / \mathrm{s}=1054.6$ watts 
NBS.114A (REV. 7.73)

\begin{tabular}{|c|c|c|}
\hline $\begin{array}{l}\text { U.S. DEPT. OF COMM. } \\
\text { BIBLIOGRAPHIC DATA } \\
\text { SHEET }\end{array}$ & $\begin{array}{l}\text { 1. PUIBI.IC ATION OR RIEPORT NO. } \\
\text { NBSIR 77-1222 }\end{array}$ & 3. Recipient's Accession No. \\
\hline \multirow{2}{*}{\multicolumn{2}{|c|}{$\begin{array}{l}\text { 4. TITLE AND SUBTITLE } \\
\text { Reproducibility of the Radiant Panel Test Method } \\
\text { (ASTM E 162-67) Using Polyurethane Foam, } \\
\text { Neoprene and Hardboard Specimens }\end{array}$}} & $\begin{array}{l}\text { 5. Publication Date } \\
\text { March } 1977\end{array}$ \\
\hline & & 6. Performing Organization Code \\
\hline \multicolumn{2}{|l|}{$\begin{array}{l}\text { 7. AUTHOR(S) } \\
\text { T. G. Lee }\end{array}$} & 8. Performing Organ. Report No. \\
\hline \multirow{2}{*}{\multicolumn{2}{|c|}{$\begin{array}{l}\text { 9. PERFORMING ORGANIZATION NAME ANI ADDRESS } \\
\text { NATIONAL BUREAU OF STANDARDS } \\
\text { DEPARTMENT OF COMMERCE } \\
\text { WASHINGTON, D.C. } 20234\end{array}$}} & $\begin{array}{c}\text { 10. Project/Task/Work Unit No. } \\
4915678\end{array}$ \\
\hline & & 11. Contract/Grant No. \\
\hline \multirow{2}{*}{\multicolumn{2}{|c|}{ 12. Sponsoring Organization Name and Complete Address (Street, City, State, ZIP) }} & $\begin{array}{l}\text { 13. Type of Report \& Period } \\
\text { Covered } \\
\text { Final Report }\end{array}$ \\
\hline & & 14. Sponsoring Agency Code \\
\hline
\end{tabular}

15. SUPPI.EMENTARY NOTES

16. ABSTRACT (A 200-word or less factual summary of most significant information. If document includes a significant bibliography or literature survey, mention it here.)

Interlaboratory evaluation of the Radiant Panel Method (ASTM E 162-67) for flame spread testing of two flexible foam and one hardboard specimens was made. Results obtained by 13 laboratories based on 4 replicate tests, showed that the between-lab coefficient of variation on the flame-spread index ( $I_{S}$ ) was $21 \%$ for Hardboard A, 35\% for Urethane B and $45 \%$ for Neoprene C. The within-lab coefficient of variation for the Hardboard was $9.9 \%$. The higher variability of results for the foam materials was caused by the rapid melting of the Urethane $B$ and unstable flame front of the Neoprene $C$ specimens during the tests. An important source of error for some laboratories was in the determination of $\beta$, the calibration constants, and the inappropriate use of base stack temperature correction. Statistics on the reproducibility of the flame-spread factor ( $F_{S}$ ), heat evolution (Q) and $I_{s}$ are also given. A new pilot burner and other modifications of the method were found useful.

17. KEY WORDS (six to twelve entries; alphabetical order; capitalize only the first letter of the first key word unless a proper name; separated by semicolons) ASTM E 162; coefficient of variation; flame spread tests; flexible polyurethane; hardboard; interlaboratory evaluation; neoprene; radiant panel test; round robin; test method.

18. AVAILABILITY Unlimited

For Official Distribution. Do Not Release to NTIS

Order From Sup. of Doc., U.S. Government Printing Office Washington, D.C. 20402, SD Cat. No.C13

[X Order lirom National Technical Information Service (NTIS) Springfield, Virginia 22ISI

\begin{tabular}{|l|c|}
\hline $\begin{array}{l}\text { 19. SECURITY CLASS } \\
\text { (THIS REPURT) } \\
\text { UNCL ASSIFIED }\end{array}$ & 21. NO. OF PAGES \\
\hline $\begin{array}{l}\text { 20. SECURITY CLASS } \\
\text { (THIS PAGE) }\end{array}$ & $\begin{array}{l}\text { 22. Price } \\
\text { UNCI.ASSIIII.I) }\end{array}$ \\
\hline
\end{tabular}

